\title{
FBX018/Circrna211/Mir-431/CSF1 Axis Regulates the Establishment of Endometrial Receptivity Though Activating MAPK and CSF1R/PI3K/AKT/Mtor Pathways in Dairy Goats
}

\section{Lichun Yang}

Northwest A\&F University: Northwest Agriculture and Forestry University

Xiaorui Liu

Northwest A\&F University: Northwest Agriculture and Forestry University

\section{Lei Zhang}

Northwest A\&F University: Northwest Agriculture and Forestry University

Danni Li

Northwest Agriculture and Forestry University College of Animal Science and Technology

Guili Li

Northwest A\&F University: Northwest Agriculture and Forestry University

Jiuzeng Cui

Northwest A\&F University: Northwest Agriculture and Forestry University

\section{Xiaopeng An}

Northwest A\&F University: Northwest Agriculture and Forestry University

\section{Binyun Cao}

Northwest A\&F University: Northwest Agriculture and Forestry University

Yu-Xuan Song ( $\square$ yuxuan_song2016@163.com )

Northwest Agriculture and Forestry University https://orcid.org/0000-0002-5302-7835

\section{Research}

Keywords: endometrial epithelium cells, receptive endometrium, circRNA211, miR-431, CSF1

Posted Date: May 13th, 2021

DOI: https://doi.org/10.21203/rs.3.rs-510867/v1

License: (c) (7) This work is licensed under a Creative Commons Attribution 4.0 International License. Read Full License 


\section{Abstract}

Background: Endometrial epithelial cells proliferation and secretion of various cytokines have a strong impact on the formation of receptive endometrium, which is known as a physiological status that allows an activated embryo to attach to the endometrium for a limited time. Circular RNAs and miRNAs can be involved in the dynamic physiological changes of endometrium by regulating relevant functional target genes in the uterus. Our work presented here with the ultimate purpose of revealing the latent molecular mechanism of FBX018/circRNA211/miR-431/CSF1 axis in the establishment of endometrial receptivity of dairy goats.

Results: In vitro, we found a regulatory network of FBX018/circRNA211/miR-431/CSF1 in goat endometrial epithelial cells that circRNA211 severed as a sponge for miR-431, resulting in weakening the inhibition of miR-431 on target genes CSF1 and FBX018. FBX018/circRNA211/miR-431/CSF1 axis promoted the proliferation through regulating the key proteins of Ras, Raf, MEK, ERK in MAPK pathway via CCK-8, EdU, flow cytometry and Western blot assays. Furthermore, FBX018/circRNA211/miR-431/CSF1 axis activated the phosphorylation of key proteins PI3K, AKT and mTOR in PI3K-mTOR pathway by CSF1R, thereby promoting the establishment of endometrial receptivity. In vivo models, mice injected with miR-431 agomir showed that the endometrial thickness and the number of pinopodes were significantly decreased by HE staining and scanning electron microscope. Immunohistochemistry results showed that VEGF and OPN proteins were down-regulated and MUC1 protein was up-regulated under the treatment of miR-431 agomir. Further study demonstrated that miR-431 inhibited embryo implantation by impeding the establishment of endometrial receptivity.

Conclusion: Ultimately, our study revealed a regulatory mechanism of FBXO18/circRNA211/miR-431/CSF1 axis in goat endometrial epithelial cells. This circRNA/miRNA/mRNA regulatory network presented here in vitro and in vivo models may provide a novel insight into the potentially regulating endometrium biological functions and promoting the formation of endometrium receptivity.

\section{Background}

The well development of endometrium is of vital importance to improve the lambing rate of dairy goats. Receptive endometrium (RE) is a status that the endometrial tissue is remodeled for allowing the embryo to attach in a limited time after a series of physiological changes [1]. The establishment of endometrial receptivity is a key prerequisite for embryo implantation and clinical evidence suggests that endometrial receptivity insufficiency resulted in $30 \%$ of embryo implantation failure in humans [2]. Therefore, it is extremely important to elucidate the molecular mechanism of the establishment of endometrial receptivity in dairy goats.

Circular RNAs (circRNAs) were first discovered from a RNA virus study by electron microscopy in 1976. CircRNAs may act as miRNA sponges to regulate the expression of miRNAs, which regulated the expression of target gene indirectly [3]. Although circRNAs have pleiotropic roles in a variety of biological processes including regulation of myoblasts differentiation in bovine [4], embryo implantation in mouse [5], and milk synthesis in goats [6, 7], but their contribution to the formation of RE remains largely unclear.

As a highly conserved small non coding RNA, microRNAs (miRNAs) are essential epigenetic regulators for gene expression. MiRNAs serve as post-transcriptional regulators that have been reported repeatedly to regulate various biological processes, such as cell proliferation [8], apoptosis $[9,10]$ and differentiation [11]. There is increasing evidence that the formation of endometrial receptivity is indeed associated with profound changes in the 
expression of epigenetic regulatory factors. For example, miR-184 promotes the expression of FOXM1 and VEGF in protein levels, which are the marker genes of RE [12]; In addition, miR-26a increased the OPN protein level and decreased $C O X-2$, which suggested that miR-26a regulate the development of endometrial receptivity in dairy goats [13].

The function of endometrial tissue has become a research hotspot in recent years. Human endometrium transcriptome has been widely used to study the complex function of endometrium, and many diagnostic markers related to endometrium receptivity have been found $[14,15]$. Previous study had identified the circRNAs and miRNAs expression profiles in the goat endometrium on the 5th day of pregnancy (pre- receptive endometrium, $\mathrm{PE}$ ) and the 15th day (receptive endometrium, RE). circRNA211 and miR-431 were differentially expressed in the endometrium, suggesting that they may be involved in the formation of endometrial receptivity $[16,17]$. It is of interest to know whether circRNA211 and miR-431 may be involved in regulating the proliferation or apoptosis of dairy goat endometrial epithelial cells (GEECs), thus contributing to the establishment of endometrial receptivity.

Abnormal expression of uterine cytokines leads to failure of embryo implantation and decrease of pregnancy rate in human $[18,19]$ and mouse $[20,21]$. For example, leukemia inhibitory factor (LIF) is mainly expressed in the endometrial epithelial cells during the receptive stage to facilitate the adhesion and implantation of blastocysts in the uterus [18]; The cytokines IGF1 and FGFs can regulate endometrial lumen epithelial cells proliferation through autocrine and paracrine action [21]. It has been reported that vascular endothelial growth factor (VEGF) derived from endometrial epithelium and glands significantly enhanced the adhesion of human endometrial epithelial cells to trophoblast extracellular matrix and promoted the growth of mouse blastocysts [22].

Colony-stimulating factor1, also known as macrophage colony-stimulating factor (CSF1/M-CSF), is a kind of cytokine that binds to CSF1 receptor (CSF1R) encoded by the $c$-fms proto-oncogene [23]. CSF1 is a key regulator of inflammation, tissue repair, regeneration and fibrosis in endometrium. Studies have shown that CSF1 supernatant can promote the growth and migration of endometrial cells in vitro and prevent their fibrosis and apoptosis [24]. However, there has been less previous evidence for the specific regulatory mechanism of CSF 1 in GEECs.

In the current study, we constructed FBXO18/circRNA211/miR-431/CSF1 regulatory network and performed a detailed analysis with quantitative real-time PCR, Western blot, Cell Counting Kit-8 (CCK-8) assay, EdU staining and Annexin V-FITC/PI apoptosis kit to elucidate dynamic changes in GEECs. Furthermore, influence of FBX018/circRNA211/miR-431/CSF1 axis on the establishment of endometrial receptivity was verified by in vivo and in vitro model experiments. These findings highlight the complicated and tightly-regulated circRNA /miRNA/mRNA network that occurs in the establishment of endometrial receptivity.

\section{Materials And Methods}

\section{Vector constructions}

For the purpose of overexpression assays, goat CSF1 CDS sequence (XM_013962557.2) and FBXO18 CDS sequence (XM_018056944.1) were cloned by polymerase chain reaction(PCR) using total RNA extracted from endometrial tissue. The two fragments were inserted into $\mathrm{pMD}^{\mathrm{TM}} 19-\mathrm{T}$ vectors (TaKaRa, Beijing, China) and sequenced entirely. Then, target fragments of CSF1 with Xho I and Kpn I restriction sites and FBXO18 with Xho I 
and BamH I restriction sites were inserted into pcDNA3.1 (+) vector (Promega, Madison, USA) by double digestion experiment. Finally, the insertion results were verified by DNA sequencing.

The complete sequence of goat circRNA211 was cloned by PCR to insert into PMD ${ }^{\text {TM }} 19-\mathrm{T}$ vectors (TaKaRa, Beijing, China). Subsequently, target fragment of circRNA211 was inserted into pcD2.1-cir vectors (Geneseed Biotech, Guangzhou, China), restriction sites were $\mathrm{Kpn}$ I and BamH I. All the insertion results were verified by DNA sequencing. Primer sequences used to construct overexpression vectors are shown in the following Table1.

Table 1

Primer sequence

\begin{tabular}{|c|c|}
\hline gene & Primer sequences $\left(5^{\prime} \rightarrow 3^{\prime}\right)$ \\
\hline \multirow[t]{2}{*}{ CSF1 } & F:CGAGGTACCATGACCGCGCGGGGCGCC \\
\hline & R:TGCTCGAGCTACACTGGCAGCTCCTCCTGTCTGTCCTTC \\
\hline \multirow[t]{2}{*}{ FBXO18 } & F:CGGGATCCATGGCCAAAAGCAATTCTGTGG \\
\hline & R:CCCTCGAGTCAGAAGACGAGGAAGAGCAGGG \\
\hline \multirow[t]{2}{*}{ circRNA211 } & F:GGGGTACCTGAAATATGCTATCTTACAGGGCATCAACGTGTGGGCC \\
\hline & R:CGGGATCCTCAAGAAAAAATATATTCACTGGCGTGCAGTCCT \\
\hline
\end{tabular}

Bioinformatics analysis showed that miR-431 had complementary binding sites with CSF1, FBXO18 and circRNA211 respectively using miRanda and Targetscan7.0. To obtain wild-type reporter vector for DualLuciferase Reporter Assay, 3'UTR of CSF1(310bp), CDS of FBXO18(259bp), full circRNA211 sequence (946bp) were cloned and inserted downstream of the Renilla luciferase gene between Xho I and Not I sites in the psiCHECKTM-2 vector (Promega, Madison, USA) respectively. Next, the mutant primers were designed, and the mutated-type vectors with mutated sites were also constructed by using Fast Mutagenesis Kit V2(Vazyme, Nanjing, China). All insertion results were verified by DNA sequencing. All the primer sequences needed are shown in Table 2. 
Table 2

Primer sequence

\begin{tabular}{|c|c|}
\hline gene & Primer sequences $\left(5^{\prime} \rightarrow 3^{\prime}\right)$ \\
\hline \multirow[t]{2}{*}{ WT-CSF1 } & F:CCCTCGAGTTCTGGGCCTGCTGGTCTG \\
\hline & R:AAGCGGCCGCACGGACAAGTGTTTGCTCTCCG \\
\hline \multirow[t]{2}{*}{ WT-circRNA211 } & F:CGCTCGAGGGCATCAACGTGTGGGCC \\
\hline & R:ATGCGGCCGCCTGGCGTGCAGTCCT \\
\hline \multirow[t]{2}{*}{ MUT-CSF1 } & F:CCAGGGTGGAGCTAAGCGCTGGCCTCTGCC \\
\hline & R:GCTTAGCTCCACCCTGGGCATCCAAGCCA \\
\hline \multirow[t]{2}{*}{ MUT-circRNA211 } & F:TCATCTGTGGAGCCTTCCACTCCATGGCCTACG \\
\hline & R:GAAGGCTCCACAGATGACGTTGCTGGGGAA \\
\hline \multirow[t]{2}{*}{ MUT-FBXO18 } & F:TCATCTGTGGAGCCTTCCACTCCATGGCCTACG \\
\hline & R:GAAGGCTCCACAGATGACGTTGCTGGGGAA \\
\hline
\end{tabular}

Note: underlined nucleotides indicate mutation sites.

\section{Cell culture}

GEECs resuscitated for culture from liquid nitrogen tanks had been isolated and purified according to the procedure as previously described [13]. The cells were cultured in a filtered DMEM/F12 medium (Gibco, CA, USA) with $10 \%$ fetal bovine serum (Gibco, CA, USA), $1000 \mathrm{U} / \mathrm{mL}$ penicillin and $100 \mathrm{ug} / \mathrm{mL}$ streptomycin at $37^{\circ} \mathrm{C}$ in a humidified atmosphere with $5 \%$ CO2. HEK293T cells were cultivated in high-glucose DMEM containing $10 \%$ fetal bovine serum, $100 \mathrm{U} / \mathrm{mL}$ penicillin and streptomycin in a humidified incubator with $5 \% \mathrm{CO} 2$ at $37^{\circ} \mathrm{C}$ to proliferate.

\section{Dual-Luciferase Reporter Assay}

HEK293T cells were cultivated in 24-well plates at a density of $60 \%$ before transfection. Then plasmids $(1 \mu \mathrm{g} / \mathrm{well})$ including the wild-type (psiCHECK2-WT) or mutated (psiCHECK2-MUT) were co-transfected with the miR-431 mimic, miR-431 inhibitors, NC, or NC inhibitors (100nM/well) synthesized by GenePharma (Shanghai, China) using Lipofectamine ${ }^{\text {TM }}$ RNAiMAX Reagent (Invitrogen, CA, USA) into 293T cells, respectively. After $36 \mathrm{~h}$, activities contained Firefly (hluc+) and Renilla (hRluc)were measured according to the manufacturer's instructions (Promega, USA) using Thermo Scientific Varioskan Flash (Thermo Fisher Scientific).

\section{RNA extraction and RT-qPCR}

GEECs were cultivating in 6-well plates at a density of 60\% before transfection. CSF1-si-RNA, circRNA211-si-RNA were also synthesized by GenePharma (Shanghai, China). Then overexpression vectors (pcDNA3.1-CSF1, pcDNA3.1-FBX018, pcDNA2.1-circRNA211), CSF1-si-RNA, circRNA211-si-RNA, miR-431 mimic, miR-431 inhibitors, $\mathrm{NC}$, and NC inhibitors were transfected respectively or co-transfected to GEECs as required. At $24 \mathrm{~h}$ later, total RNA in GEECs was extracted using Trizol reagent (TaKaRa, Dalin, China). In this study, circRNA211 was incubated with $3 \mathrm{U} / \mu \mathrm{g}$ RNase $\mathrm{R}$ at $37^{\circ} \mathrm{C}$ for 15 min before reverse transcription into cDNA. Reverse transcription reaction was performed using the Prime Script RT reagent Kit with gDNA Eraser (TaKaRa, Dalian, China) based on the manufacturer's instructions. Subsequently, the mRNA expression levels of different genes were quantified using 
SYBR Green PCR MasterMix (TaKaRa, Dalian, China) in a $20 \mu l$ reactions. PCR reaction program was carried out according to the manufacturer's instructions. The date was collected through the CFX Connect Real-Time PCR Detection System (Bio-Rad, CA, USA). $2^{-\triangle \Delta C t}$ was used to analyze the relative mRNA expression in GEECs, $\beta$-actin or U6 mRNA level was used for normalization. qPCR primer sequences are shown in Table 3.

Table 3

Primer sequence for $\mathrm{qPCR}$

\begin{tabular}{|c|c|c|}
\hline gene & $\begin{array}{l}\text { GenBank } \\
\text { accession No. }\end{array}$ & Primer sequences $\left(5^{\prime} \rightarrow 3^{\prime}\right)$ \\
\hline \multirow[t]{2}{*}{$\beta$-actin } & \multirow[t]{2}{*}{ XM_018039831.1 } & F: GATCTGGCACCACACCTTCT \\
\hline & & R: GGGTCATCTTCTCACGGTTG \\
\hline \multirow[t]{2}{*}{ CSF1 } & \multirow[t]{2}{*}{ XM_013962557.2 } & F: CCATGAGAGGCAGCACAAGGAAG \\
\hline & & R: GCCAGCAAGACCAGGATGATACTG \\
\hline \multirow[t]{2}{*}{ FBX018 } & \multirow[t]{2}{*}{ XM_018056944.1 } & F: TGAAGAGCAAGCCGTCAACAAGG \\
\hline & & R: GAGGTTCAGCACGCACAGGTC \\
\hline \multirow[t]{2}{*}{ circRNA211 } & \multirow[t]{2}{*}{ / } & F: GAAGTTGGGCGAGTGCAAGG \\
\hline & & R: CCAAGGCCCACACGTTGATG \\
\hline \multirow[t]{2}{*}{ U6 } & \multirow[t]{2}{*}{ / } & F: CTCGCTTCGGCAGCACA \\
\hline & & R: AACGCTTCACGAATTTGCGT \\
\hline $\begin{array}{l}\text { miR-431- } \\
\text { Loop }\end{array}$ & / & GTCGTATCCAGTGCAGGGTCCGAGGTATTCGCACTGGATACGACGCATGAC \\
\hline $\begin{array}{l}\operatorname{miR}-431- \\
\text { FW }\end{array}$ & / & GCGCGCTGTCTTGCAGGCC \\
\hline $\begin{array}{l}\text { Reverse } \\
\text { Primer }\end{array}$ & / & GTGCAGGGTCCGAGGT \\
\hline
\end{tabular}

\section{Protein extraction and Western blot (WB)}

GEECs were cultured in 6-well plates at a density of 50\% when transfection. Then pcDNA3.1- CSF1, pcDNA3.1FBX018, pcDNA2.1-circRNA211, CSF1-si-RNA, circRNA211-si-RNA, miR-431 mimic, miR-431 inhibitors, NC, and NC inhibitors were transfected respectively or co-transfected to GEECs as required. $48 \mathrm{~h}$ later, GEECs were collected using RIPA (Radio Immunoprecipitation Assay, Solarbio, Beijing, China) lysis buffer and phenylmethanesulfonyl fluoride (PMSF, Solarbio, Beijing, China). Protein extraction and WB analysis methods have been described in detail by previous researchers $[13,25]$. Proteins were visualised using enhanced chemiluminescence luminous fluid (Amersham, GE Healthcare Lifesciences) through gel documentation system (Biospectrum 410, UVP). Finally, proteins were quantified using Quantity One program (Bio-Rad, CA, USA). All antibodies used in this study are given in Table 4. 
Table 4

Antibody information for Western blot

\begin{tabular}{|c|c|c|}
\hline Name & Manufacturer & Product Number \\
\hline$\beta$-actin & Beyotime, Shanghai, China & AA128 \\
\hline CSF1 & BBI, Shanghai, China & D163065 \\
\hline BAX & BBI, Shanghai, China & D120073 \\
\hline BCL2 & BBI, Shanghai, China & D191052 \\
\hline RAS & Gene Tex, America & GTX132480 \\
\hline p-RAF1(Ser642) & BBI, Shanghai, China & D151385 \\
\hline RAF1 & BBI, Shanghai, China & D220484 \\
\hline p-MEK1(Ser298) & Abways, Shanghai, China & CY5854 \\
\hline MEK1 & Abways, Shanghai, China & CY5168 \\
\hline p-ERK1/2(Thr202/Tyr204) & Cell Signaling, America & \#9101 \\
\hline ERK $1 / 2$ & Cell Signaling, America & \#9102 \\
\hline CSF1R & BBI, Shanghai, China & D260600 \\
\hline P-CSF1R(Phospho-Tyr809) & BBI, Shanghai, China & D151408 \\
\hline PI3K p110 beta & Bioss, Beijing, China & bs-6423R \\
\hline p-PI3K p110 beta (Ser1070) & Bioss, Beijing, China & bs-6417R \\
\hline AKT & Cell Signaling, America & \#9272 \\
\hline p-AKT (Ser473) & Cell Signaling, America & \#9271 \\
\hline mTOR & Abways, Shanghai, China & CY5306 \\
\hline p- mTOR (S2448) & Abways, Shanghai, China & CY6571 \\
\hline HRP-labeled Goat Anti-Rabbit IgG $(\mathrm{H}+\mathrm{L})$ & Beyotime, Shanghai, China & A0208 \\
\hline HRP-labeled Goat Anti-Mouse IgG $(\mathrm{H}+\mathrm{L})$ & Beyotime, Shanghai, China & A0216 \\
\hline
\end{tabular}

\section{Cell proliferation assays}

GEECs viability were evaluated using Cell Counting Kit-8 (CCK-8, ZETA ${ }^{\mathrm{TM}}$ Life, USA). GEECs were cultured in 96-well plates at a density of $2 \times 10^{3}$ cells/well in a 100 $\mu \mathrm{L}$ volume, then pcDNA3.1-CSF1, pcDNA3.1-FBX018, pcDNA2.1circRNA211, CSF1-si-RNA, circRNA211-si-RNA, miR-431 mimic, miR-431 inhibitors, NC, and NC inhibitors were transfected respectively or co- transfected to GEECs as required. $24 \mathrm{~h}$ later, $10 \mu \mathrm{L} \mathrm{CCK8}$ solution was added to each well of the plate (Do not introduce bubbles to the well, as they interfere with the absorbance reading). Then 96-well plates were put into cell incubator again and incubated for 2 hours in darkness. Subsequently, the absorbance was calculated at 450nm using an epoch microplate reader (Biotek, Winooski, USA). 
GEECs proliferation were evaluated using the 5-Ethynyl-2'-deoxyuridine (EdU, Ribobio, Guangzhou, China) in 48well plates with a $200 \mu \mathrm{L}$ volume. After $24 \mathrm{~h}$ transfection, $200 \mu \mathrm{L}$ cell culture medium with a final concentration of $50 \mu \mathrm{M}$ EdU was added to each well for the first staining and incubated for $2 \mathrm{~h}$. Thereafter, after washed in PBS three times GEECs were treated with DAPI dying $15 \mathrm{~min}$ at $37^{\circ} \mathrm{C}$ for the second staining (Refer to the manufacturer's instructions for the detailed steps). Images were captured by inverted fluorescence microscope. Cell proliferation was evaluated by calculating EdU staining cells (EdU positive cells)/DAPI staining cells (total cells) ratio.

\section{Cell apoptosis analysis}

GEECs apoptosis were evaluated using Annexin V-FITC/PI apoptosis kit (Liankebio, Hangzhou, China). Before transfection, GEEC $_{S}$ were cultured to $60 \%$ density in 6-well plates. After $24 \mathrm{~h}$ transfection, All the cells in each well, including in the culture supernatant, were collected into a $10 \mathrm{~mL}$ centrifuge tube, and then added $250 \mu \mathrm{L} 1 \times$ binding buffer, $5 \mu \mathrm{L}$ FITC, 10 $\mu \mathrm{L}$ PI successively following the protocol of the manufacturer. Apoptosis positive cells were obtained through the flow cytometry method (FCM).

\section{Scanning electron microscopy (SEM)}

Uterus samples were collected when the left uterine horn was injected with miR-431 agomir and the other side was used as negative control. Firstly, samples were fixed with $2.5 \%$ glutaraldehyde fixed solution at $4{ }^{\circ} \mathrm{C}$ for more than $6 \mathrm{~h}$. Then, $0.1 \mathrm{M} / \mathrm{L}$ PBS with pH7.2 was flushed every 5 minutes, rinse for 6 times in total. Subsequently, samples were progressively dehydrated in an alcohol concentration gradient solution, followed by $2 \%$ isoamyl alcohol for $3 \mathrm{~h}$ and freeze drying. Samples were then painted with silver conductive plastic using a vacuum coating apparatus. Finally, samples were observed and photographed under the JSM-6330F SEM (JEOL, Japan).

\section{Hematoxylin-eosin (HE) staining}

Mouse endometrial tissue was fixed with $4 \%$ paraformaldehyde and then embedded in paraffin. Then sections were stained with hematoxylin for $5 \mathrm{~min}$ and eosin for $1 \mathrm{~min}$. After dehydration, the sections were sealed with neutral resin. The morphological features and endometrial thickness of mice were observed under inverted microscope (Nikon, Japan).

\section{Immunohistochemistry (IHC)}

The paraffin sections were immersed in 3\% hydrogen peroxide solution for $15 \mathrm{~min}$, then closed with $3 \%$ bovine serum albumin and incubated at room temperature for $20 \mathrm{~min}$. Then, the primary antibody $(1: 200)$ was added at $4^{\circ} \mathrm{C}$ overnight (the specific antibody information is shown in Table 5). Biotin labeled secondary antibodies were incubated at $37^{\circ} \mathrm{C}$ for 20 min. Finally, freshly prepared DAB chromogenic agent and hematoxylin were added. Positive cells are stained in brown and hematoxylin is stained in blue. Inverted microscope was used for observation (Nikon, Japan). 
Table 5

Antibody information for immunohistochemistry

\begin{tabular}{|lll|}
\hline Name & Manufacturer & Product Number \\
\hline VEGF & Abways, Shanghai, China & CY5096 \\
\hline OPN & Abways, Shanghai, China & CY5333 \\
MUCl & $\mathrm{BBI}$, Shanghai, China & D262100 \\
\hline LIF & $\mathrm{BBI}$, Shanghai, China & D260555 \\
\hline
\end{tabular}

\section{Statistical analysis}

IBM SPSS Statistics 26.0 (IBM, USA) was used to process data. The results are showed as means \pm standard error (SE) by one-way ANOVA and the LSD (Least Significant Difference) test. Differences are considered significant at ** $P<0.01$ or * $P<0.05$.

\section{Results}

\section{MiR-431 induced the apoptosis of GEECs in vitro}

Previous studies have reported the first miRNA expression profile in goat endometrium during embryo implantation related to the PE and RE [16]. Depending on the sequencing data, miR-431 was highly differential expression in goat endometrium during embryo implantation. Hence, miR-431 was invoked as a candidate miRNA to investigate its role in goat endometrial epithelial cells (GEECs). Figure 1A showed the transfection efficiency of miR-431 mimic in GEECs by stem-loop RT-qPCR. The results revealed that the mRNA expression level of miR-431 was remarkably increased compared with NC, which indicated that miR-431 could be used in the further experiments (Fig. 1A).

To further investigate the role of miR-431 in GEECs, we conducted CCK-8 and EdU assays. The CCK-8 assay revealed that miR-431 mimics significantly inhibited the viability of GEECs compared with NC. Nevertheless, there was no noteworthy difference between miR-431 inhibitor and NCH (Fig. 1B). The EdU assays also showed that miR-431 mimics notably suppressed the proliferation of GEECs while miR-431 inhibitor exhibited the opposite result (Fig. 1C).

Furthermore, Annexin V-FITC/PI apoptosis kit combined with FCM was used to estimate GEECs apoptosis. The results indicated that the number of apoptosis cells increased in miR-431 mimics group, but decreased in miR-431 inhibitor group (Fig. 1D). Similarly, we also performed the WB analysis to assess the protein of apoptosis-related, $B C L 2$ and BAX. The results revealed that miR-431 mimics reduced the BCL2 protein level strikingly while increased the expression of BAX, whereas miR-431 inhibitor exhibited the opposite result (Fig. 1E). Altogether, these results demonstrated that miR-431 induced the apoptosis of GEECs in vitro.

\section{MiR-431 targeted CSF1 in GEECs}

As a post-transcriptional regulator of gene expression, miRNA plays a biological role in degrading mRNA of target genes or inhibiting translation [26]. Then we wondered whether miR-431 induces cell apoptosis by negatively 
regulating its target gene. And we utilized the TargetScan and miRanda to screen CSF1 as the target gene of miR431.

We constructed wild-type plasmid contained 3'UTR of CSF1(about 310bp) and mutated-type plasmid with mutated sites of CSF1 3'UTR for dual-luciferase reporter assay in HEK293T cells (Fig. 2A). As showed in the Fig. 2B, luciferase activity of miR-431 mimics co-transfection with wild-type plasmid was markedly lower compared with the NC group. However, there was no significant difference between miR-431 mimics and NC group after co-transfection with mutant plasmid (Fig. 2B). Together, the results revealed that miR-431 reduced the expression of CSF1 by binding with its complementary sequences.

To further verify the targeting effect of miR-431 for CSF1, we implemented RT-qPCR and Western blot experiment. Consistently, the results showed that overexpression miR-431 mimics dramatically depressed the expression of CSF1 in mRNA and protein level while miR-431 inhibitor had the reverse results (Fig. 2C-D). Subsequently, we tested the transfection efficiency of CSF1 in the level of mRNA and protein in GEECs. The results revealed that pc3.1-CSF1 significantly increased the CSF1 expression in mRNA and protein levels. However, miR-431 cotransfection with CSF1 reduced the expression of CSF1 protein, suggesting that the miR-431 targeted CSF1 (Fig. 2E-F). However, CSF1 with small interference RNA (si-CSF1) decreased the expression of CSF1 in mRNA and protein levels.

\section{CSF1 promoted the proliferation of GEECs in vitro}

To determine the targeted regulation effect of 431 on CSF1, we used CCK-8 and EdU assays to ascertain the function of CSF1 in GEECs. The results indicated that overexpression CSF1 elevated cell vitality while cotransfection of miR-431 abolished this stimulating effect. Similarly, we found that after interfering with the expression of $C S F 1$, the proliferative effect was removed (Fig. 3A-B). Additionally, we sought to test whether overexpression CSF1 declined the apoptosis rate in GEECs. The results of Annexin V-FITC/PI staining presented that pc3.1-CSF1 significantly decreased the number of apoptotic cells. However, miR-431 co-transfection with CSF1 somewhat removed the effect of CSF1 on GEECs apoptosis (Fig. 3C). And the WB results also strongly support this assertion that CSF1 inhibited cell apoptosis. Conversely, silencing of CSF1 with small interference RNA induced cell apoptosis, which could weaken the trend when co-transfection with miR-431 inhibitor (Fig. 3D). Collectively, these findings suggested that CSF1 promoted cell proliferation and miR-431 induces cell apoptosis through negative regulation of CSF1.

\section{MiR-431 inhibited MAPK signaling pathway through CSF1}

Previous studies have reported that Ras/Raf/MEK/ERK signaling pathway plays a critical role in cell proliferation and cell apoptosis $[6,7,27,28]$. It has also been reported that CSF1 can induce monocytes to produce VEGF through SP1/MAPK/ERK signaling pathway, thus promoting in vivo angiogenesis [29]. Consequently, we speculated that miR-431 may induce GEEC apoptosis through Ras/Raf/MEK/ERK signaling pathway, on account of its negative regulation on CSF1 expression. Subsequently, we treated Western blot to evaluate the expression levels of Ras, Raf, MEK and ERK proteins and their phosphorylated proteins under the treatment of miR-431 and/or CSF1. The results indicated that miR-431 and interference CSF1(si-CSF1) both significantly decreased the expression of Ras, P-Raf, P-MEK and P-ERK proteins (Fig. 4A, 4C). However, overexpression of CSF1 observably increased the expression of Ras, $\mathrm{p}-\mathrm{MEK}$, and $\mathrm{p}$-ERK proteins. When miR-431 was co-transfection with CSF1, the effect of CSF1 on MAPK signal transduction pathway was eliminated to a certain extent (Fig. 4B). Those findings 
suggested that CSF1 may active the MAPK cascade pathway, while miR-431 inhibit that via down-regulating CSF1.

\section{Correlation analysis of circRNA211 and FBX018 expression in GEECs}

Increasingly evidences indicated that circRNAs, as molecular sponges of miRNAs, can further regulate the expression of downstream target genes [30,31]. According to the expression profile of circular RNA library constructed in the laboratory and bioinformatics analysis, circRNA211 containing the binding site of miR-431 seed sequence was selected for further study. Meanwhile, sequencing results showed that FBXO18 (F-box protein, Helicase, 18), which also has targeted binding sites of miR-431 in the CDS region through bioinformatics prediction, was the host gene of circRNA211 [17]. The transfection efficiency of FBXO18, circRNA211 and small interfering RNA of circRNA211 (si-circRNA211) shown in Fig. 5A demonstrated that they can be utilized in subsequent experiments (Fig. 5A). Furthermore, we used RT-qPCR to determine the relationship between circRNA211 and FBX018. The results showed that high expression of FBXO18 could enhance the expression of circRNA211 (Fig. 4B), whereas circRNA211 interference had no effect on the expression of FBXO18 (Fig. 4C). In summary, all the above results suggested that the expression of circRNA211was positively correlated with FBX018.

\section{CircRNA211 competitively inhibited miR-431}

Subsequently, full circRNA211 sequence and mutated sequence of circRNA211 with mutated sites was cloned and inserted into the psiCHECKTM-2 vector to construct wild type plasmid (WT-circRNA211) or mutated-type plasmid (MUT-circRNA211) for dual-luciferase reporter assay. The results showed that relative luciferase activity in miR-431 mimics co-transfection with WT-circRNA211 plasmid was dramatically lower than the NC group in HEK293T cells. Whereas, miR-431 mimic co-transfection with MUT-circRNA211 plasmid had no effect on the relative luciferase activity (Fig. 5D). RT-qPCR showed that the increasing expression of circRNA211 could significantly reduce the expression of miR-431 at the mRNA level. On the contrary, interfering circRNA211 markedly raised the expression of miR-miR-431 (Fig. 5E). Therefore, all the results suggested that circRNA211 served as a sponge for miR-431 in GEECs, thus inhibiting the expression of miR-431.

\section{MiR-431 targeted FBX018 in GEECs}

Furthermore, we constructed vectors including the binding site of FBXO18 and miR-431 (WT-FBXO18) or mutant fragment of $F B X 018$ (MUT-FBX018) to detect luciferase activity in HEK293T cells. The results revealed that the luciferase activity of miR-431 mimics co-transfection with WT-FBX018 plasmid was greatly declined compared with the NC group. Nonetheless, miR-431 mimics co-transfection with MUT-FBXO18 plasmid had no effect on the relative luciferase activity (Fig. 5F). In addition, miR-431 mimics also sharply declined the expression of $F B X 018$, while miR-431 inhibitor increased that at the mRNA level (Fig. 5G). Therefore, miR-431 targeted FBXO18 and inhibited the expression of FBX018 in GEECs.

\section{Effects of circRNA211and FBX018 on CSF1 expression}

To further confirm whether circRNA211 could serve as competitive endogenous RNAs (ceRNAs), we use RT-qPCR and WB to examine the expression levels of CSF1 in GEECs. The results showed that overexpression of circRNA211 significantly increased the mRNA and protein levels of CSF1 (Fig. 6A), while si-circRNA211 decreased that (Fig. 6C). Meanwhile, overexpression FBX018 also greatly enhanced the expression of CSF1 in the mRNA and 
protein levels (Fig. 6B), which indicated that FBX018 also facilitated the expression of CSF1 in GEECs. Overall, these results demonstrated that circRNA211 and FBXO18 can promote the expression of CSF1 in GEECs.

\section{CircRNA211 and FBX018 promoted the proliferation of GEECs}

To investigate the biological role of circRNA211 and FBXO18 in GEECs, cell proliferation was evaluated using CCK-8 kit. The results showed that overexpression of circRNA211 and FBXO18 increased cell activity, and this increase was reversed while circRNA211 or FBX018 co-transfection with miR-431 (Fig. 7A, C). On the contrary, after circRNA211 interference, the cell viability decreased dramatically (Fig. 7B). The results of EdU staining also showed that the increase in FBXO18 or circRNA211 considerably promoted cell proliferation, while interference circRNA211 inhibited cell proliferation (Fig. 7D-F). Moreover, Annexin V-FITC/PI staining results showed that FBX018 and circRNA211 remarkably reduced the apoptosis of GEECs. Whereas, overexpression of miR-431 eliminated this decrease effect on the apoptosis of GEECs to some extent (Fig. 7G, I). Furthermore, si-circRNA211 elevated the number of apoptosis cells (Fig. 7H). On the other hand, the results of WB also showed that overexpression of FBXO18 and circRNA211 promoted cell proliferation (Fig. 7J, L), while si-circrNA211 induced cell apoptosis (Fig. 7K). Generally, those results manifested that circRNA211 and FBXO18 promote the proliferation of GEECs.

\section{CircRNA211 and FBX018 activated MAPK signaling pathway by inhibiting miR-431}

Similarly, we also conducted WB assay to examine the effect of circRNA211 and FBXO18 on MAPK signaling pathway. The results indicated that overexpression of circRNA211 activated the activity of Ras protein, which in turn activated the phosphorylation of downstream Raf and MEK proteins (Fig. 8A). Overexpression of FBXO18 can also activate the activity of Ras protein, and then activated protein phosphorylation of downstream Raf, MEK and ERK successively (Fig. 8C). However, activity of phosphorylated proteins on the MAPK signaling pathway was inhibited under si-circRNA211 treatment (Fig. 8B). Altogether the results showed that circRNA211 and FBXO18 may promote the proliferation of GEECs by activating MAPK signaling pathway due to their 'sponge' effect on miR-431.

\section{FBX018 /circRNA211/miR-431/ CSF1 axis promoted the formation of endometrial receptivity in dairy goats}

Western blot was used to investigate the effects of FBX018/circRNA211/miR-431/CSF1 regulatory axis on CSF1R/PI3K/ AKT /mTOR signaling pathway. Firstly, we analyze the effect of miR-431 on the expression of CSF1R, PI3K, AKT and mTOR proteins and their phosphorylated proteins. The results showed that miR-431 mimics notably reduced the phosphorylation level of CSF1R protein. Interestingly enough, miR-431 further inhibited the proteins of PI3K, AKT and mTOR from phosphorylating. Conversely, miR-431 inhibitor increased the protein phosphorylation level of CSF1R, PI3K, and AKT (Fig. 9A).

In addition, the effects of CSF1 on the expression of PI3K, AKT, mTOR and their phosphorylated proteins were also detected. WB results indicated that overexpression CSF1 promoted the phosphorylation of CSF1R protein, which in turn activated the PI3K/AKT/mTOR signaling pathway. However, the activation effect in PI3K/AKT/mTOR signaling pathway was alleviated when CSF1 co-transfection with miR-431 mimics (Fig. 9B). Meanwhile, after the expression of CSF1 was interfered (si-CSF1), the ratios of CSF1R, PI3K, and AKT proteins and their phosphorylated proteins decreased (Fig. 9C). 
Through the verification of targeted relationship, we found that circrNA211 promoted the expression of CSF1 via sponging miR-431. Therefore, it is speculated that circrNA211 triggered the PI3K/mTOR/AKT signaling pathway by inducing CSF1R protein phosphorylation. Western blot results revealed that circrNA211 obviously enhanced the ratio of P-CSF1R/CSF1R, P-PI3K/PI3K, P-AKT/AKT and P-mTOR/ mTOR protein levels. After co-transfection with circRNA211, miR-431 reduced the phosphorylation levels of CSF1R, PI3K and AKT proteins to a certain extent (Fig. 10A). On the contrary, interference with circRNA211 (si-circRNA211) sharply decreased the phosphorylation level of CSF1R, thereby inhibiting the activation of the PI3K/AKT/mTOR pathway (Fig. 10B). Similarly, as the host gene for circrNA211, FBX018 also elevated the expression levels of P-CSF1R and P-AKT proteins (Fig. 10C).

Therefore, the $F B X 018 /$ circRNA211/miR-431/CSF1 regulatory axis can regulate the establishment of endometrial receptivity of dairy goats through the CSF1R/PI3K/ AKT/mTOR signaling pathway.

\section{MiR-431 inhibited the formation of endometrial receptivity of mice in vivo}

The endometrium of the mice reached receptive status on the 4th day of pregnancy, and embryo implantation occurred on the 5th day [32]. Firstly, RT-qPCR was performed to detect the expression level of miR-431 in endometrial tissues. Compared with the negative control (NC) on the right, the expression level of miR-431 in the left uterine horn was prominently increased after miR-431 agomir injection (Fig. 11A). In order to investigate the effect of miR-431 on the receptive period of the uterus in mice, the surface morphology of the endometrium on the 4th day of pregnancy was observed by scanning electron microscopy (SEM). The results suggested that the pinopodes in the NC group were abundant and well-developed, which in the miR-431 agomir group were few and dysplastic (Fig. 11B). HE staining showed that the endometrial thickness of the NC group was noticeably thicker than that of the miR-431 group (Fig. 11C).

Pinopodes is a morphological marker of endometrial receptivity, while some genes, such as VEGF, LIF, OPN and $P R L$, can be considered as molecular markers [33]. Therefore, immunohistochemistry was used to detect the expression of marker genes related to endometrial receptivity in mice at the 4th day of gestation. The results showed that VEGF and OPN proteins in the miR-431 agomir group were significantly lower than those in the NC group, while the LIF protein had no significant change in the miR-431 agomir group and the NC group. Whereas, under the treatment of miR-431 agomir, MUC1 protein was notably higher than that in the NC group (Fig. 11D and Table 6). Moreover, the number of embryos attached to the endometrium is drastically reduced in miR-431 agomir group compared to NC (Fig. 11E). Taken together, miR-431 may inhibit embryo implantation by impeding the establishment of endometrial receptivity. 
Table 6

Immunohistochemistry staining results of receptivity marker genes in mouse endometrium

\begin{tabular}{|c|c|c|c|c|}
\hline Marker gene & Index & Area & IOD & AOD \\
\hline \multirow[t]{2}{*}{ VEGF } & NC & $82677.0 \pm 17156.2$ & $32694.8 \pm 5483.3$ & $0.313 \pm 0.0010$ \\
\hline & miR-431 agomir & $135532.3 \pm 18223.8$ & $38873.2 \pm 5271.9$ & $0.287 \pm 0.0002^{\star \star}$ \\
\hline \multirow[t]{2}{*}{$O P N$} & NC & $91895.3 \pm 29141.8$ & $24496.2 \pm 7774.3$ & $0.266 \pm 0.0025$ \\
\hline & miR-431 agomir & $77638.7 \pm 6137.7$ & $18209.6 \pm 1389.8$ & $0.235 \pm 0.0005^{\star \star}$ \\
\hline \multirow[t]{2}{*}{ MUC1 } & NC & $120249 \pm 7142.8$ & $34132.4 \pm 1745.9$ & $0.284 \pm 0.0018$ \\
\hline & miR-431 agomir & $45602.3 \pm 5184.6$ & $14579.7 \pm 1695.8$ & $0.320 \pm 0.0006^{\star \star}$ \\
\hline \multirow[t]{2}{*}{ LIF } & NC & $37969 \pm 10623.9$ & $11222.5 \pm 2752.0$ & $0.300 \pm 0.0104$ \\
\hline & miR-431 agomir & $11538.3 \pm 2523.6$ & $3774.7 \pm 775.3$ & $0.328 \pm 0.0039$ \\
\hline
\end{tabular}

Area was the positive area, IOD was integrated optical density, AOD $=$ IOD/Area. Date are expressed as mean $\pm S E$, $\mathrm{n}=3$, ** indicated the differences were very significant $(P<0.01)$.

\section{Discussion}

Successful embryo implantation requires a cross talk between receptive endometrium and activated blastocyst so that the dynamic physiological changes of endometrium are crucial for the successful implantation of embryos [34]. However, the establishment of endometrial receptivity is a complex and dynamic process that more study is needed to explore the precise mechanism of endometrial receptivity.

To further elucidate the role of microRNAs in the endometrium of dairy goats during embryo implantation, we first explored the biological function of miR-431 in GEECs. In this work, we illustrated that miR-431 inhibited the cell proliferation with CCK-8 and EdU assays, and miR-431 inhibition had the opposite effect. On the contrary, overexpression miR-431 increased the number of apoptosis cells while decreased the ratio of BCL2/BAX protein levels. The above results suggested that miR-431 had an inhibitory effect on the proliferation of endometrial epithelial cells in dairy goats.

In this study, it is urgent for us to focus on miR-431 downstream target gene, thus exploring the molecular mechanism of miR-431 inhibiting cell proliferation in dairy goats. There have three reasons for us to choose CSF1 for the further research in the following: (1) Bioinformatics analysis showed that 3'UTR of CSF1 gene has a targeted binding site with the seed sequence of miR-431, suggesting CSF1 is one of the target genes of miR-431. (2) CSF1 is a marker gene for endometrial receptivity $[35,36]$. (3) The CSF1/CSF1R axis plays a vital role in the communication between endometrial epithelial cells and surrounding cells [37].

Next, we probed how miR-431 exerted its epigenetic regulation through the downstream target gene. Our work suggested that miR-431 targeted CSF1 in HEK293T cells and suppressed the expression of CSF1 on mRNA and protein levels in GEECs. The expression of CSF1 in mRNA and protein levels was reduced significantly under the treatment of small interfering RNA of CSF1 (si-CSF1). Subsequently, the effects of CSF1 on the proliferation and apoptosis of endometrial epithelial cells in dairy goats were also examined. The results of CCK-8 and EdU presented that the si-CSF1 distinctly inhibited cell proliferation. However, overexpression of CSF1 visibly promoted 
cell proliferation. Interestingly, miR-431 alleviated the effect of CSF1 on cells to some extent when miR-431 was co-transfected with CSF1. In addition, si-CSF1 acutely boosted the number of apoptotic cells in GEECs while CSF1 overexpression decreased that.

Previous studies found that when GEECs were in the proliferative state, it was beneficial to the establishment of endometrial receptivity of dairy goats [33]. The expression level of CSF1 protein in human endometrium during pregnancy is much higher than that in non-pregnancy period, and the content of the placenta is also high [38]. Extensive evidence suggests that maternal uterus has immune rejection and inflammatory reaction when embryo implantation. As an anti-inflammatory cytokine, the high expression of CSF1 can reduce the inflammatory response of endometrium and promote the survival of endometrial epithelial cells, thus facilitating embryo implantation [39]. Therefore, CSF1, as a molecular marker of endometrial receptivity, may promote the establishment of endometrial receptivity by promoting the proliferation and survival of GEECs.

In recent years, BAX and BCL2 proteins have been found to be closely related to cell apoptosis, and the BCL2/BAX ratio is regarded as one of the markers of clinical tumor prognosis [40]. Consistently, our data suggested that both miR-431 and si-CSF1 decreased the ratio of BCL2/BAX, while CSF1 increased the ratio of BCL2/BAX. And coexpression of miR-431 with CSF1 showed a neutral effect partly. Furthermore, Western blot results showed that overexpression of miR-431 or interference of CSF1 significantly decreased the protein levels of Ras, P-Raf, P-MEK and P-ERK. Whereas, the proteins of Ras, P-MEK and P-ERK were up-regulated under the treatment of CSF1 overexpression. Hence, the above results suggested that miR-431 may block the MAPK pathway via downregulating CSF1, thereby inhibiting the proliferation of GEECs. In contrast, CSF1 promoted the proliferation of GEECs by activating the MAPK signaling pathway.

The next aim of this study was to further confirm the regulation of circRNA /miRNA/mRNA networks in the endometrium of dairy goats, thus we turned our attention to miR-431 upstream regulator. Recently, several circRNAs and microRNAs involved in endometrial physiological changes have been identified in goat endometrium. For instance, circRNA-9119 promoted the expression of PTGS2 by sponging miR-26a in GEECs [41]; circRNA8073 sponged miR-449a to regulate the development of endometrial receptivity [42]. CircRNAs are known to play a pivotal role in the regulation of gene expression by sponging miRNAs, but their contribution to the formation of endometrium receptivity remains to be further elucidated. In our previous study, we found that circRNA211 and miR-431 were expressed differentially in the endometrium of dairy goats during embryo implantation, suggesting it may have an enormous effect on the endometrial development $[16,17]$. Therefore, the precise roles of circRNA211 and miR-431 in goat uterus need to be further studied.

Although $F B X 018$ is a host gene for circRNA211, its regulation and biological role in goat endometrium remain unknown. Here, we performed RT-qPCR to ascertain the relationship between circRNA211 and FBXO18 in GEECs. Interestingly, circRNA211 was up-regulated when FBXO18 was highly expressed, whereas interference with circRNA211 (si-circRNA211) barely affected the expression of FBX018. This study suggested that circRNA211 competitively inhibited miR-431 and further suppressed its mRNA level. Nevertheless, the expression of miR-431 was up-regulated after interference with circRNA211.

In addition, we confirm that miR-431 targeted CDS of FBXO18 and inhibited its expression at mRNA level, indicating that miR-431 targets $F B X 018$. Based on the above results, we speculated that the overexpression FBX018 in GEECs could be splicing into rings through some unknown pathway, thus promoting the generation of circRNA211.

Page $15 / 32$ 
As the previous study reported that two RNAs are mutually ceRNAs that have the same microRNA recognition elements (MREs) and can competitively bind miRNAs. RNAs sharing multiple MREs are considered to be a kind of 'RNA language', which can form a ceRNAs network to make mRNA crosstalk effectively [43, 44]. To further validate the regulatory relationship on circRNA211, FBXO18 and CSF1, the expression level of CSF1 was detected by RT-qPCR and WB. The result showed that circRNA211 and FBXO18 markedly increased the expression of CSF1 via miR-431.

To further investigate the mechanism of circRNA211 and FBXO18 regulating cell physiological activities, we used CCK-8 and EdU staining to evaluate cell viability, and performed Annexin V-FITC/PI staining combined with flow cytometry to detect the apoptosis rate of GEECs. The results indicated that overexpression of circRNA211 and FBX018 promoted cell proliferation, while si-circRNA211 inhibited cell proliferation. Indeed, co-transfection of miR431 with circRNA211 or $F B X 018$ greatly attenuated the promotion effect of circRNA211 and $F B X 018$ on cell proliferation. These findings further supported the idea that circRNA211 served as miR-431 sponge or FBXO18 promote cell proliferation via inhibiting the expression of miR-431.

The purpose of this research was to ascertain the signaling pathways that regulated cell proliferation and apoptosis through the FBXO18/circRNA211/miR-431/CSF1 axis. There was substantial evidence demonstrating that MAPK signaling pathway is involved in cell physiological activity $[6,7,27,28]$. In this study, overexpression circRNA211 or FBX018 activated Ras proteins, which in turn triggered downstream phosphorylation levels of Raf, MEK, and ERK proteins. Nevertheless, activation of phosphorylated proteins was blocked under si-circRNA211 treatment. These results suggested that circRNA211 may promote the proliferation of GEECs by activating the MAPK signaling pathway due to its "sponge" effect on miR-431. Previous results have shown that miR-431 inhibited the activation of the MAPK signaling pathway by targeting CSF1. Therefore, the results of this study suggested that the $F B X 018 /$ circRNA211/miR-431/CSF1 regulatory axis can promote the proliferation of GEECs through the MAPK signaling pathway. Previous studies have shown that the expression of proliferation cell nuclear antigen (PCNA) in RE was significantly higher than that in PE, which suggested that the endometrial epithelium cells in the state of proliferation is conducive to the establishment of endometrium receptivity in dairy goats [33]. Together these results provide important insights for us that FBXO18/circRNA211/miR-431/CSF1 axis promoted the proliferation of GEECs through the MAPK signaling pathway, thus facilitating the establishment of endometrial receptivity of dairy goats.

To provide further understanding on the establishment of endometrial receptivity, we performed Western blot to analyze the expression of related proteins. In our work, the phosphorylation levels of CSF1R, PI3K and AKT proteins were significantly down-regulated in miR-431 and si-CSF1 group, while the phosphorylation levels of CSF1R, PI3K, AKT and mTOR proteins were significantly up-regulated after the overexpression of CSF1. Similarly, overexpression of circRNA211 and FBXO18 activated the phosphorylated protein of CSF1R, which activated the downstream PI3K/AKT/mTOR pathway successively. Conversely, the CSF1R/PI3K/AKT/mTOR signaling pathway was inhibited by si-circRNA211.

Accumulating evidence indicates that uterine cytokines profoundly influence on the pregnancy success and embryo development through mediating maternal uterus to embryo dialogue[45]. Ligand/receptor-mediated signaling is the main mechanism by which CSF1 acts. Cytokine of CSF1 can activate the phosphorylation of CSF1 receptor (CSF1R) protein, which triggered downstream PI3K/AKT/mTOR signaling pathway [35]. Our work found that CSF1, as a marker gene for endometrial receptivity, play an active role in the establishment of 
endometrial receptivity by activating the PI3K/AKT/mTOR signaling pathway. Consistent with these findings, we speculated that $F B X 018 /$ circRNA211/miR-431/CSF1 regulatory axis may promote the formation of endometrial receptivity through the activation of CSF1R/ PI3K/AKT/mTOR signaling pathway.

Whereas, further research is needed to explore the function of miR-431 on endometrial physiology in pregnant mice in vivo. Here, we used mice injected with miR-431 agonists to demonstrate the effect of miR-431 for the first time. Compared with the negative control group, mice injected with miR-431 agomir showed that the thickness in endometrium and the number of pinopodes on the endometrial surface were significantly decreased. Studies have shown that endometrial epithelial pinopodes is a morphological marker of the establishment of endometrial receptivity [46]. Zhang found that the pinopodes of dairy goats also appeared only on the 15th day of gestation $(\mathrm{RE})$, but not on the 5th day (PE). Moreover, the expression levels of VEGF, OPN, LIF and PRL, as markers of endometrial receptivity, are much higher in RE than PE in the endometrium of dairy goats [33]. IHC results further suggested that VEGF and OPN proteins were down-regulated and MUC1 protein was up-regulated, while no significant changes in LIF protein was observed after miR-431 agomir injected. Even more interesting is that miR431 agonists also inhibited mouse embryo implantation compared with NC.

Studies found that $O P N$, as the ligand of integrin $\mathrm{aV} \beta 3$, was positively correlated with the expression of integrin $\mathrm{aV} \beta 3$ in endometrial tissues. LIF regulated the expression of OPN, while MUC1 antagonized integrin aV $\beta 3$. Therefore, aVB3, OPN, MUC1 and LIF played a coordinating role in the establishment of endometrial receptivity [47]. However, as an anti-adhesion molecule, MUC1 inhibit the adhesion effect between endometrial epithelial cells and embryonic trophoblast cells during embryo implantation, suggesting that it is beneficial to embryo implantation by down-regulating MUC1 expression [48]. Together, these results demonstrated that miR-431 inhibited embryo implantation by impeding the establishment of mouse endometrial receptivity. In vivo models revealed that the physiological changes of miR-431 on receptive endometrium were consistent with miR-431 inhibiting cell proliferation of GEECs in vitro, suggesting that miR-431 may inhibit the establishment of endometrial receptivity in dairy goats.

\section{Conclusions}

In the current study, we found a regulatory network of FBX018/circRNA211/miR-431/CSF1 in GEECs, in which circRNA211 severed as a sponge for miR-431, thereby weakening the inhibition of miR-431 on target genes CSF1 and FBX018. The FBX018/circRNA211/miR-431/CSF1 regulatory network promoted the proliferation of GEECs through the MAPK signaling pathway and activated the CSF1R/PI3K/AKT/mTOR signaling pathway. In vivo, miR431 inhibited embryo implantation by impeding the establishment of mouse endometrial receptivity. This study set out ultimately elucidated the importance of FBX018/circRNA211/miR-431/CSF1 axis in the establishment of endometrial receptivity of dairy goats.

\section{Abbreviations}

RE: Receptive endometrium, circRNAs: Circular RNAs, miRNAs: MicroRNAs, PE: Pre- receptive endometrium, GEECs: Dairy goat endometrial epithelial cells, LIF: Leukemia inhibitory factor, VEGF: Vascular endothelial growth factor, OPN: Osteopontin, MUC1: Mucin antigen 1, CSF1: Colony-stimulating factor1, CSF1R: Colony-stimulating factor1 receptor, FBX018: F-box protein, Helicase, 18, CCK-8: Cell counting kit-8, PCR: Polymerase chain reaction, RT-qPCR: Real-time quantitative PCR, WB: Western blot, PMSF: Phenylmethanesulfonyl fluoride, EdU: 5-Ethynyl-2'- 
deoxyuridine, FCM: Flow cytometry method, SEM: Scanning electron microscopy, HE: Hematoxylin-eosin, IHC: Immunohistochemistry, ceRNAs: Competitive endogenous RNAs, OD: Optical density, PCNA: Proliferating cell nuclear antigen.

\section{Declarations}

\section{Acknowledgements}

Not applicable.

\section{Funding}

This work was supported by the Project of Cooperating to Build Jinchang Meat Sheep Experimental Demonstration Base A289021806 (K3320219064), the China Postdoctoral Science Foundation (2019M653776, 2020M673516), the National Key Research and Development Program of China (2016YFD0500508), the Natural Science Foundation of Shaanxi Province, China (2020JQ-265), and the PhD research startup foundation of Northwest A\&F University, China (00400/Z109021811).

\section{Availability of data and materials}

All data analyzed or presented in this study are available from the corresponding author on request.

\section{Author's contributions}

LCY, DNL and LGL mainly conducted the experiments. LCY analyzed the data and drafted the manuscript. LZ and YXS revised the manuscript. XRL conceived and designed the experiments. JZC, XPA and BYC helped to collect animal samples. All authors read and approved the manuscript.

\section{Ethics approval and consent to participate}

All experimental animal care and protocols were performed depending on national and institutional guidelines and were approved by the Institutional Animal Care and Use Committee of College of Animal Science and Technology, Northwest A\&F University, China.

\section{Consent for publication}

Not applicable.

\section{Competing interests}

All authors declare that they have no competing interests.

\section{Author details}

College of Animal Science and Technology, Northwest A\&F University, Yangling, Shaanxi, 712100, P.R. China,

\section{References}


1. Craciunas L, Gallos I, Chu J, Bourne T, Quenby S, Brosens JJ, et al. Conventional and modern markers of endometrial receptivity: a systematic review and meta-analysis. Human Reproduction Update. 2019;25(2):202-23.

2. Wang X, Wu SP, Demayo FJ. Hormone Dependent Uterine Epithelial-Stromal Communication for Pregnancy Support. Other. 2017; 60(Suppl 1).

3. Kristensen LS, Andersen MS, Stagsted LVW, Ebbesen KK, Kjems J. The biogenesis, biology and characterization of circular RNAs. Nature Reviews Genetics. 2019; 20(7).

4. Peng S, Song C, Li H, Cao X, Ma Y, Wang X, et al. Circular RNA SNX29 Sponges miR-744 to Regulate Proliferation and Differentiation of Myoblasts by Activating the Wnt5a/Ca(2+) Signaling Pathway. Mol Ther Nucleic Acids. 2019;16:481-93.

5. Zhang S, Ding YB, He JL, Zhang JJ, Liu XQ, Chen XM, et al. Altered expression patterns of circular RNAs between implantation sites and interimplantation sites in early pregnant mice. J Cell Physiol. 2019;234(6):9862-72.

6. Liu Y, Hou J, Zhang M, Seleh-Zo E, Wang J, Cao B, et al. circ-016910 sponges miR-574-5p to regulate cell physiology and milk synthesis via MAPK and PI3K/AKT-mTOR pathways in GMECs. Journal of Cellular Physiology. 2020; 235(5).

7. Zhu C, Jiang Y, Zhu J, He Y, An X. CircRNA8220 Sponges MiR-8516 to Regulate Cell Viability and Milk Synthesis via Ras/MEK/ERK and PI3K/AKT/mTOR Pathways in Goat Mammary Epithelial Cells. Animals. 2020;10(8):1347.

8. Shi S, Zhou X, Li J, Zhang L, Chu G. MiR-214-3p Promotes Proliferation and Inhibits Estradiol Synthesis In Porcine Granulosa Cells. 2020.

9. Fernandez S, Risolino M, Mandia N, Talotta F, Soini Y, Incoronato M, et al. miR-340 inhibits tumor cell proliferation and induces apoptosis by targeting multiple negative regulators of p27 in non-small cell lung cancer. Oncogene. 2015;34(25):3240-50.

10. Liu Y, Hou J, Zhang M, Seleh-Zo E, Wang J, Cao B, et al. circ-016910 sponges miR-574-5p to regulate cell physiology and milk synthesis via MAPK and PI3K/AKT-mTOR pathways in GMECs. Wiley-Blackwell Online Open. 2019; 235(5).

11. Peng S, Song C, Li H, Cao X, Chen H. Circular. RNA SNX29 sponges miR-744 to inhibit proliferation and promote differentiation of myoblasts by activating the Wnt5a/Ca2 + signaling pathway. Molecular Therapy Nucleic Acids. 2019; 16.

12. Cui J, Liu X, Yang L, Che S, Guo H, Han J, et al. MiR-184 Combined with STC2 Promotes Endometrial Epithelial Cell Apoptosis in Dairy Goats via RAS/RAF/MEK/ERK Pathway. Genes (Basel). 2020; 11(9).

13. Zhang L, Liu X, Liu J, Ma X, Zhou Z, Song Y, et al. miR-26a promoted endometrial epithelium cells (EECs) proliferation and induced stromal cells (ESCs) apoptosis via the PTEN-PI3K/AKT pathway in dairy goats. J Cell Physiol. 2018;233(6):4688-706.

14. Zhang D, Sun C, Ma C, Dai H, Zhang W. Data mining of spatial-temporal expression of genes in the human endometrium during the window of implantation. Reproductive Sciences. 2012;19(10):1085-98.

15. Bhagwat SR, Chandrashekar DS, Ruchi K, Sravanthi D, Bajpai AK, Sumeet N, et al. Endometrial Receptivity: A Revisit to Functional Genomics Studies on Human Endometrium and Creation of HGEx-ERdb. Plos One. 2013;8(3):e58419-. 
16. Song Y, An X, Zhang L, Fu M, Peng J, Han P, et al. Identification and Profiling of microRNAs in Goat Endometrium during Embryo Implantation. Plos One. 2015;10(4):e0122202.

17. Song Y, Zhang L, Liu X, Niu M, Cui J, Che S, et al. Analyses of circRNA profiling during the development from pre-receptive to receptive phases in the goat endometrium. Journal of Animal Science and Biotechnology. $2019 ; 10$.

18. Salamonsen LA, Evans J, Nguyen HPT, Edgell TA. The Microenvironment of Human Implantation: Determinant of Reproductive Success. Am J Reprod Immunol. 2016;75(3):218-25.

19. Chuprinenko LM, Slavinsky AA, Sevostyanova ES, Verevkin AA, Krutova VA. Expression of vascular endothelial growth factor $a$ in the endometrium in uterine factor infertility. Kuban Scientific Medical Bulletin. 2020;27(1):85-95.

20. Evans NKB, G JDK, L.A. S NJH. Endometrial signals improve embryo outcome: functional role of vascular endothelial growth factor isoforms on embryo development and implantation in mice. Human Reproduction. 2014(10): p. 10.

21. Yan Y, Adam W, Li F, Yu W, Hong Z, Ivy Z, et al. Heparan Sulfate Proteoglycan Sulfation Regulates Uterine Differentiation and Signaling during Embryo Implantation. Endocrinology. 2018(6): p. 6.

22. Hannan NJ, Paiva P, Meehan KL, Rombauts LJF, Gardner DK, Salamonsen LA. Analysis of Fertility-Related Soluble Mediators in Human Uterine Fluid Identifies VEGF as a Key Regulator of Embryo Implantation. Endocrinology. 2011(12): p. 12.

23. Cannarile MA, Weisser M, Jacob W, Jegg A-M, Ries CH, Ruettinger D. Colony-stimulating factor 1 receptor (CSF1R) inhibitors in cancer therapy. Journal for Immunotherapy of Cancer. 2017; 5.

24. Liu D. J W, G Z, P J, M S, H, et al. CSF1-associated decrease in endometrial macrophages may contribute to Asherman's syndrome. American Journal of Reproductive Immunology. 2020; 83(1).

25. Zhang L, Liu X, Liu J, Zhou Z, Song Y, Cao B, et al. miR-182 aids in receptive endometrium development in dairy goats by down-regulating PTN expression. PLoS One. 2017;12(7):e0179783.

26. Hammond MS. An overview of microRNAs. Adv Drug Deliv Rev. 2015;87:3-14.

27. Liu X, Zhang L, Yang L, Cui J, Song Y. miRa/c induce caprine endometrial epithelial cell apoptosis by regulating circ73/ CEP55 via the RAS/RAF/MEK/ERK and PI3K/AKT/mTOR pathways. Journal of Cellular Physiology. 2020(1).

28. Lei, Zhang, Xiaorui, Liu, Sicheng C, et al. Endometrial Epithelial Cell Apoptosis Is Inhibited by a ciR8073miR181a-Neurotensis Pathway during Embryo Implantation. Molecular Therapy Nucleic Acids; 2019.

29. Laoui D, Van Overmeire E, De Baetselier P, Van Ginderachter JA, Raes G. Functional relationship between tumor-associated macrophages and macrophage colony-stimulating factor as contributors to cancer progression. Front Immunol. 2014;5(5):489.

30. Mengge $\mathrm{H}$, Zhenyu, Zhong, Mengxin Lv, et al. Comprehensive analysis of differentially expressed profiles of IncRNAs and circRNAs with associated co-expression and ceRNA networks in bladder carcinoma. Oncotarget. 2016.

31. Gu X, Li M, Jin Y, Liu D, Wei F. Identification and integrated analysis of differentially expressed IncRNAs and circRNAs reveal the potential ceRNA networks during PDLSC osteogenic differentiation. Bmc Genetics. 2017;18(1):100. 
32. Cha J, Sun X, Dey SK. Mechanisms of implantation: strategies for successful pregnancy. Nat Med. 2012;18(12):1754-67.

33. Zhang L, Liu XR, Han P, Zhou ZQ, Cao BY, Song YX. The study of endometrium at gestational days 5 and 15 in dairy goats (Capra hircus). Czech Journal of Animal Science. 2017;62(9):358-67.

34. Evans J, Rai A, Nguyen HPT, Poh QH, Elglass K, Simpson RJ, et al. Human Endometrial Extracellular Vesicles Functionally Prepare Human Trophectoderm Model for Implantation: Understanding Bidirectional MaternalEmbryo Communication. Proteomics. 2019;19(23):17.

35. Stanley ER, Chitu V. CSF-1 Receptor Signaling in Myeloid Cells. Cold Spring Harb Perspect Biol. 2014;6(6):a021857-7.

36. Mettler L, Schmutzler AG, Koch K, Schollmeyer T, Salmassi A. Identification of the M-CSF receptor in endometriosis by immunohistochemistry and RT-PCR. 2015; 52(5): 298-305.

37. Lim HJ, Dey SK. HB-EGF: A unique mediator of embryo-uterine interactions during implantation. Exp Cell Res. 2009;315(4):619-26.

38. Daiter E, Pampfer S, Yeung YG, Barad D, Stanley ER, Pollard JW. Expression of colony-stimulating factor-1 in the human uterus and placenta. J Clin Endocrinol Metab. 1992;74(4):850-8.

39. Robertson SA, Chin PY, Femia JG, Brown HM. Embryotoxic cytokines-Potential roles in embryo loss and fetal programming. J Reprod Immunol. 2018;125:80-8.

40. Velinovic M, Jankovic R, Jovanovic D, Trifunovic VS, Gavrilovic D, Stojsic J, et al. Tumor characteristics, expressions of ERCC1, Bax, p53, IGF1R, Bcl2, Bcl2/Bax and prognostic factors for overall survival in patients with lung carcinoid. Journal of Buon. 2019;24(1):256-66.

41. Zhang L, Liu X, Che S, Cui J, Liu Y, An X, et al. CircRNA-9119 regulates the expression of prostaglandinendoperoxide synthase 2 (PTGS2) by sponging miR-26a in the endometrial epithelial cells of dairy goat. Reproduction Fertility Development. 2018;30(12):1759-69.

42. Liu X, Zhang L, Liu Y, Cui J, Che S, An X, et al. Circ-8073 regulates CEP55 by sponging miR-449a to promote caprine endometrial epithelial cells proliferation via the PI3K/AKT/mTOR pathway. Biochim Biophys Acta Mol Cell Res. 2018;1865(8):1130-47.

43. Karreth FA, Tay Y, Perna D, Ala U, Pandolfi PP. In vivo identification of tumor- suppressive PTEN ceRNAs in an oncogenic BRAF-induced mouse model of melanoma. Cell. 2011;147(2):382-95.

44. Salmena L, Poliseno L, Tay Y, Kats L, Pandolfi PP. A ceRNA hypothesis: the Rosetta Stone of a hidden RNA language? Cell. 2011;146(3):353-8.

45. Klonos E, Katopodis P, Karteris E, Papanikolaou E, Tarlatzis B, Pados G. Endometrial changes in estrogen and progesterone receptor expression during implantation in an oocyte donation program. Experimental therapeutic medicine. 2020;20(6):178-8.

46. Bentin-Ley U, Sjogren A, Nilsson L, Hamberger L, Larsen JF, Horn T. Presence of uterine pinopodes at the embryo-endometrial interface during human implantation in vitro. Hum Reprod. 1999;14(2):515.

47. Xu S, Li J, Chen X, Liu B. In Vitro Study on the Regulation of Annexin IV and VEGF by hCG in the Human Endometrium. Biochem Res Int. 2020; 2020: p. 8892930.

48. Liang J, Cao D, Zhang X, Liu L, Tan Q, Shi S, et al. miR-192-5p suppresses uterine receptivity formation through impeding epithelial transformation during embryo implantation. Theriogenology. 2020;157:360-71. 


\section{Figures}
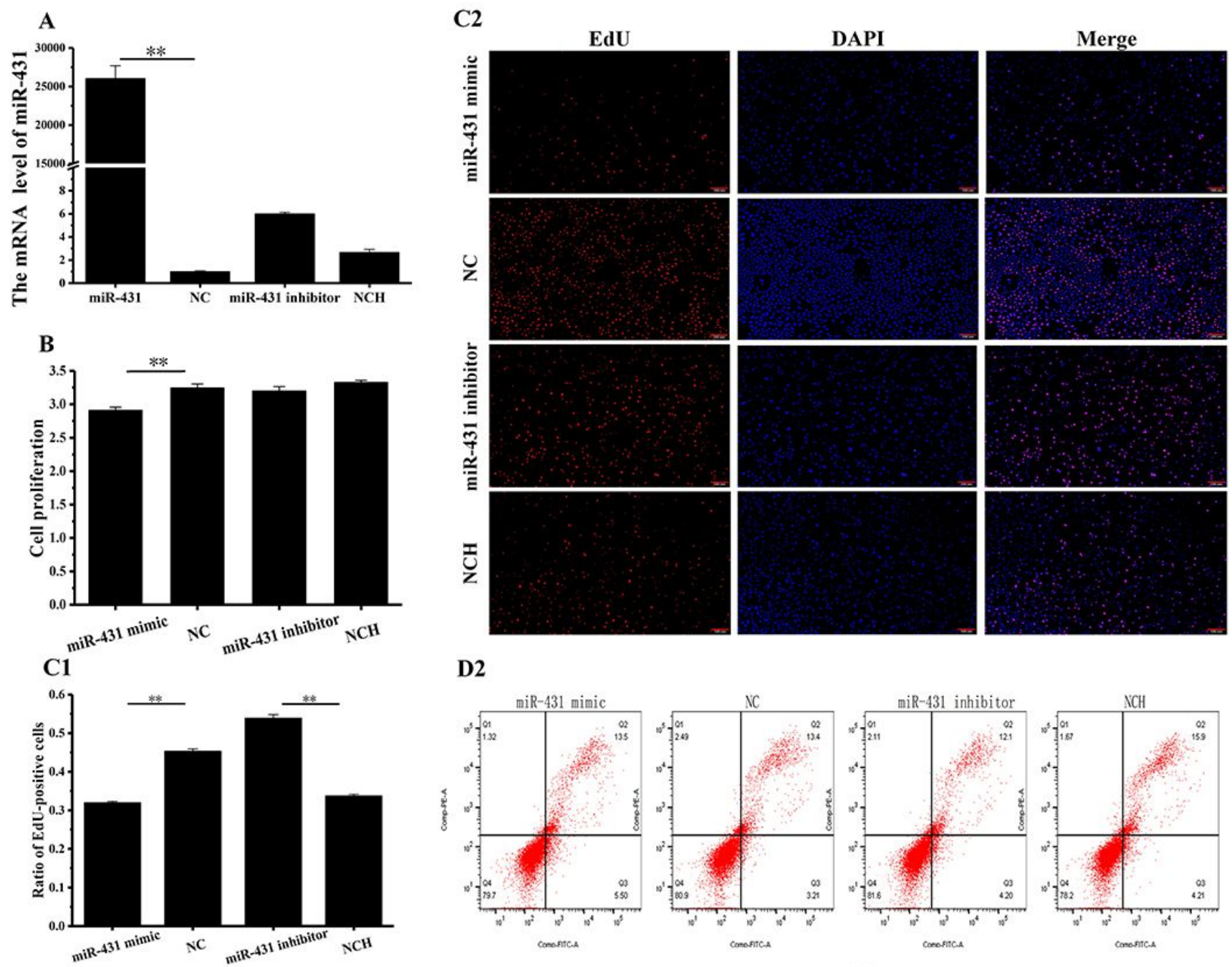

D2
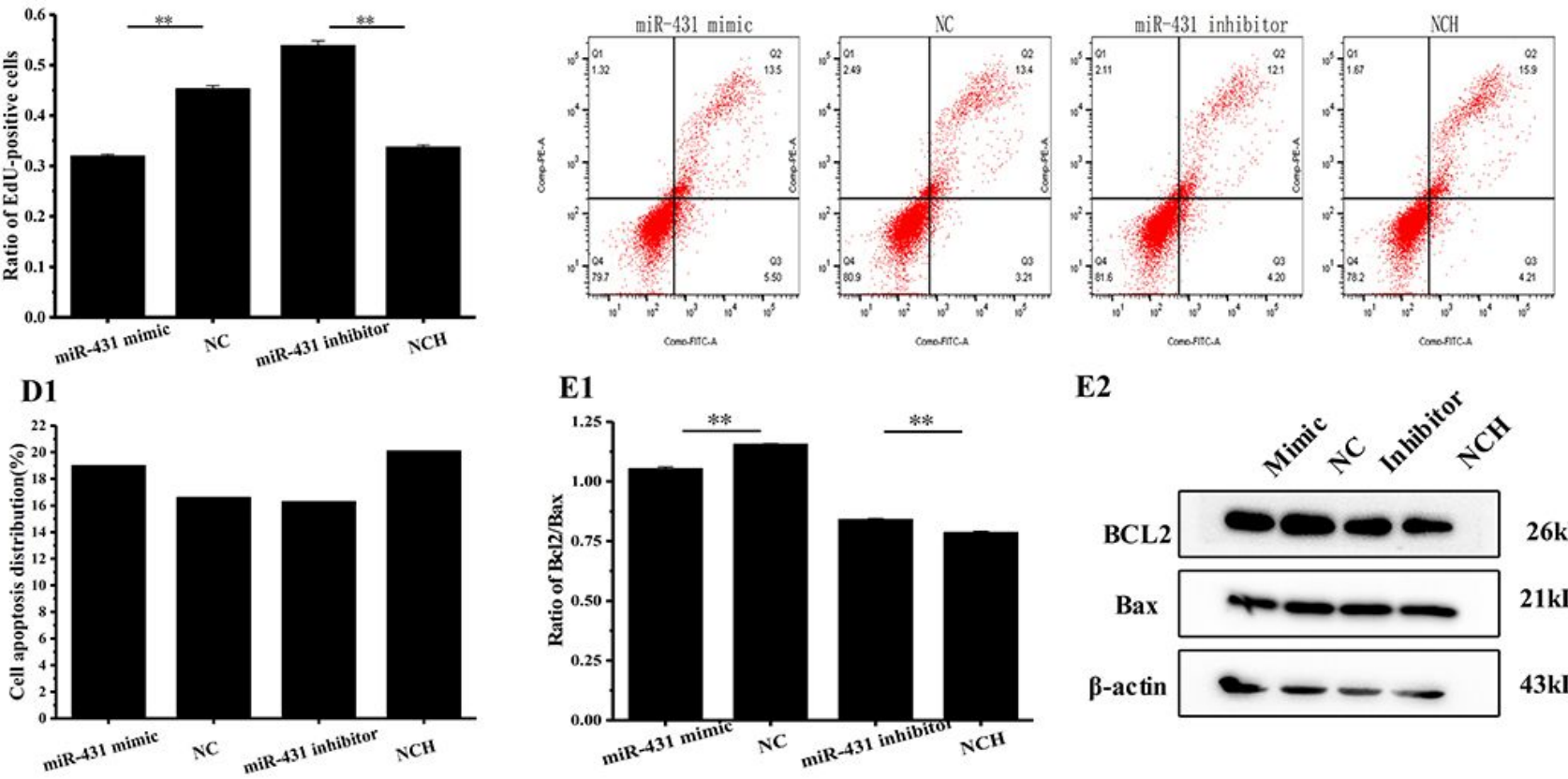

E1

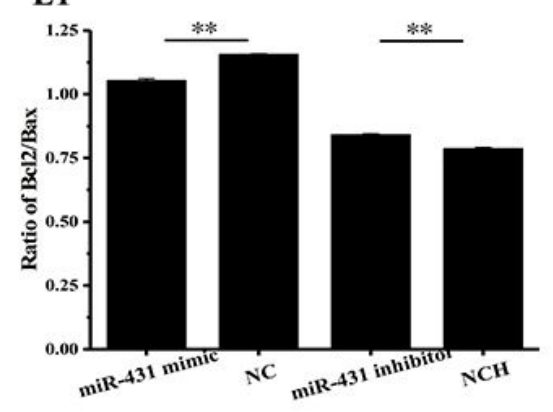

E2

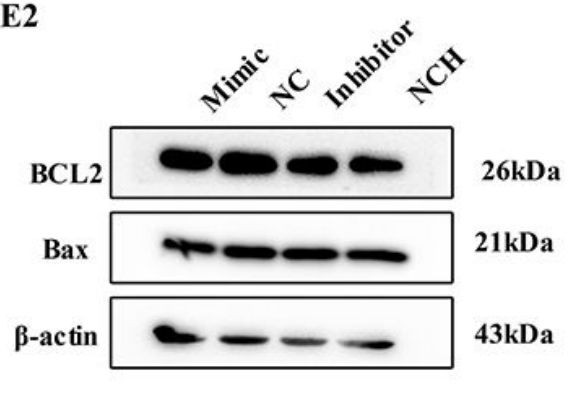

\section{Figure 1}

MiR-431 induced GEECs apoptosis. A: Transfection efficiency of miR-431 in GEECs, B: Cell proliferation was detected by CCK-8, C: Cell proliferation was detected by EdU, the scale bar was $200 \mu \mathrm{m}$, D: Cell apoptosis was detected by flow cytometry, E: Protein level was detected by Western blot, $\beta$-actin was used as a loading control. Date are expressed as mean $\pm S E, n=3$. * denotes that the difference is significant $(P<0.05)$, ** denotes that the difference is very significant $(P<0.01)$. 
A

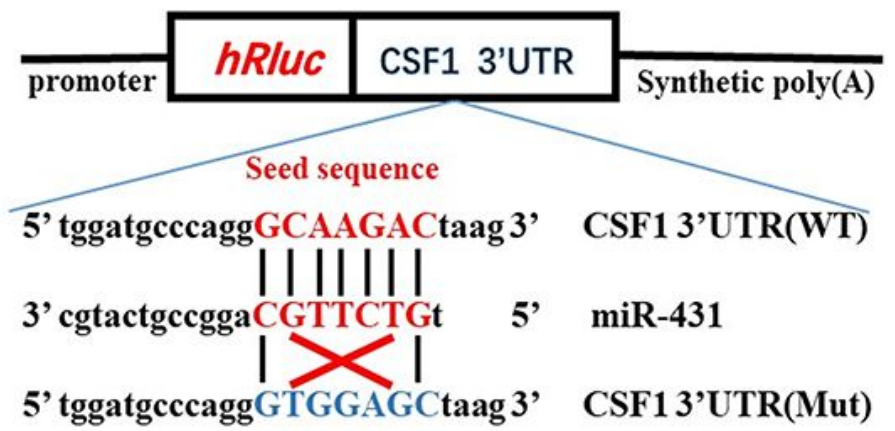

B
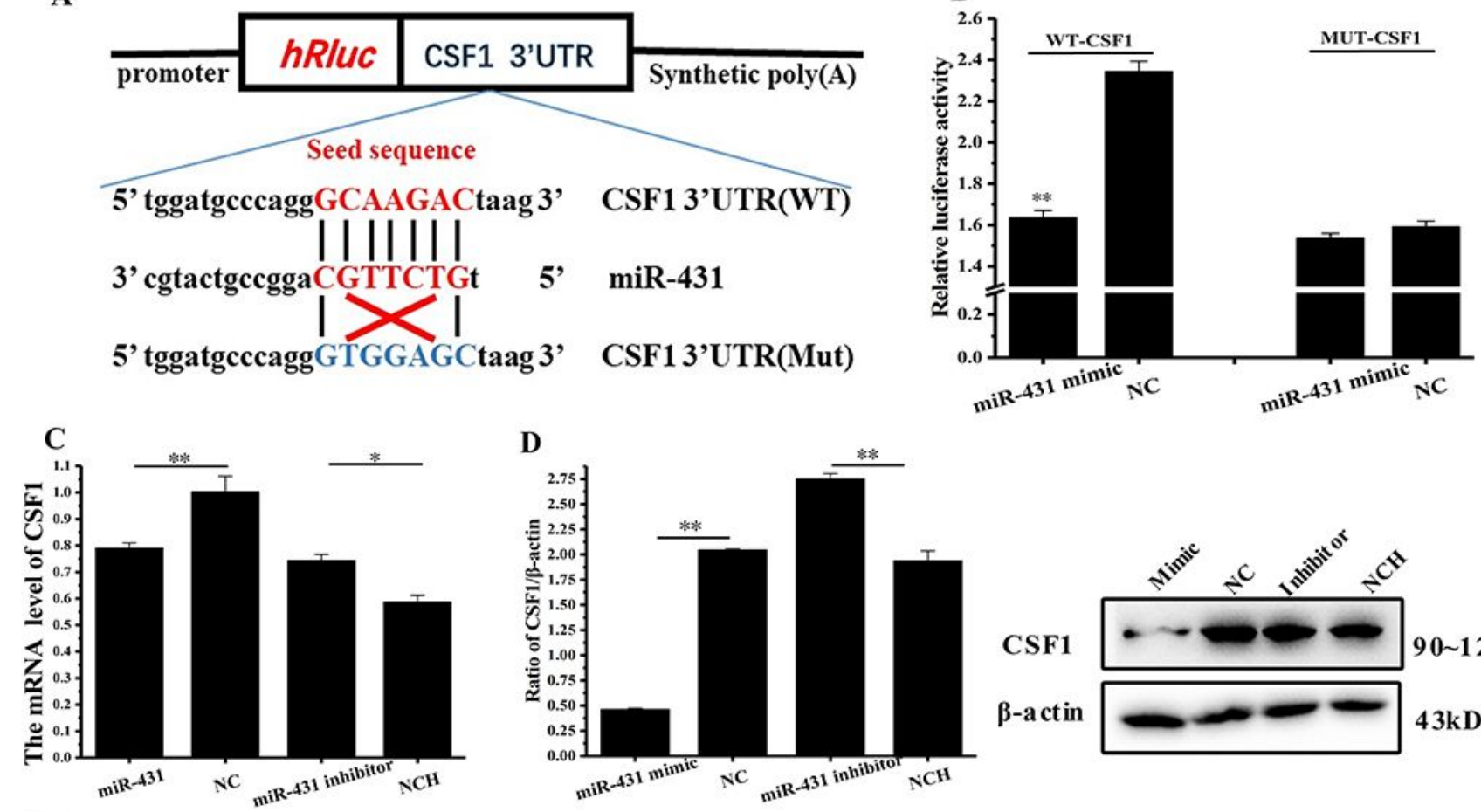

F1
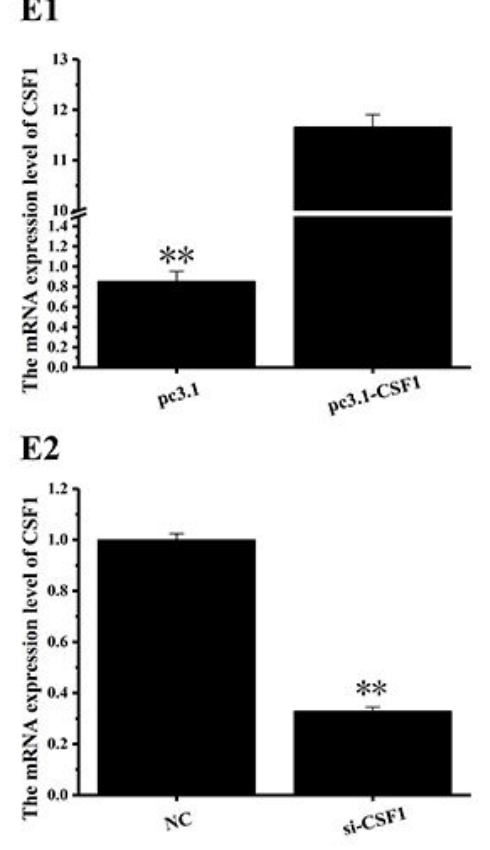
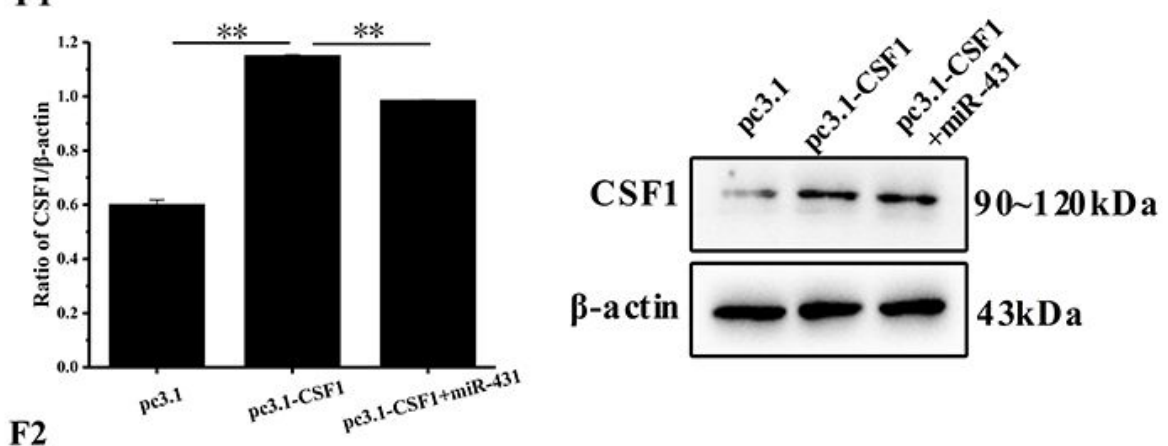

F2
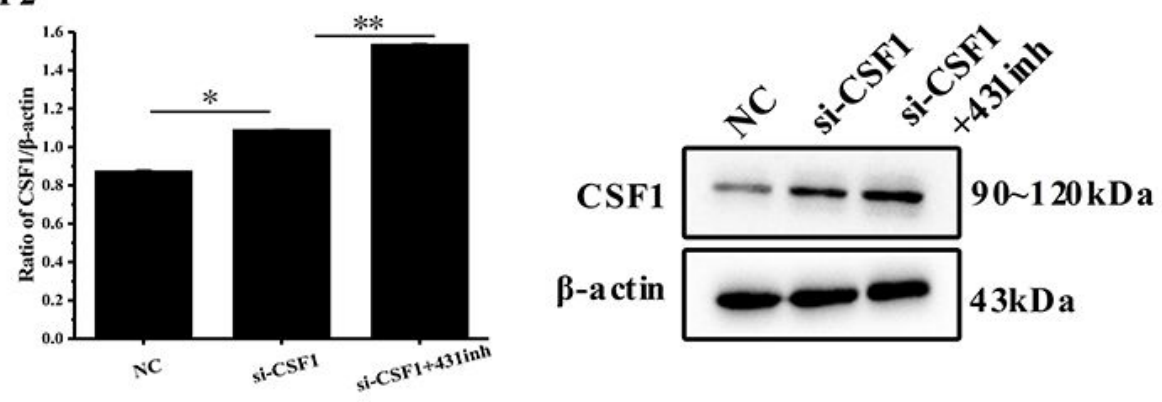

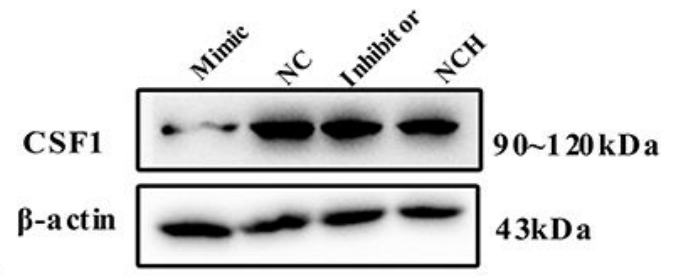

\section{Figure 2}

miR-431 inhibited the expression of CSF1. A: Schematic diagram of construction of wild-type and mutant dual luciferase reporter vectors. The nucleotide sequence marked in red is the seed sequence that miR-431 binds to CSF1, while the nucleotide sequence marked in blue is the mutant sequence. B: Detection of dual luciferase activity. C: CSF1 mRNA level was detected by RT-qPCR. D: CSF1 protein level was detected by Western blot. E: The expression level of CSF1 mRNA was detected by RT-qPCR. F: The expression level of CSF1 protein was detected by Western blot, $\beta$-actin was used as a loading control. Date are expressed as mean $\pm S E, n=3$. * denotes that the difference is significant $(P<0.05)$, ** denotes that the difference is very significant $(P<0.01)$. 


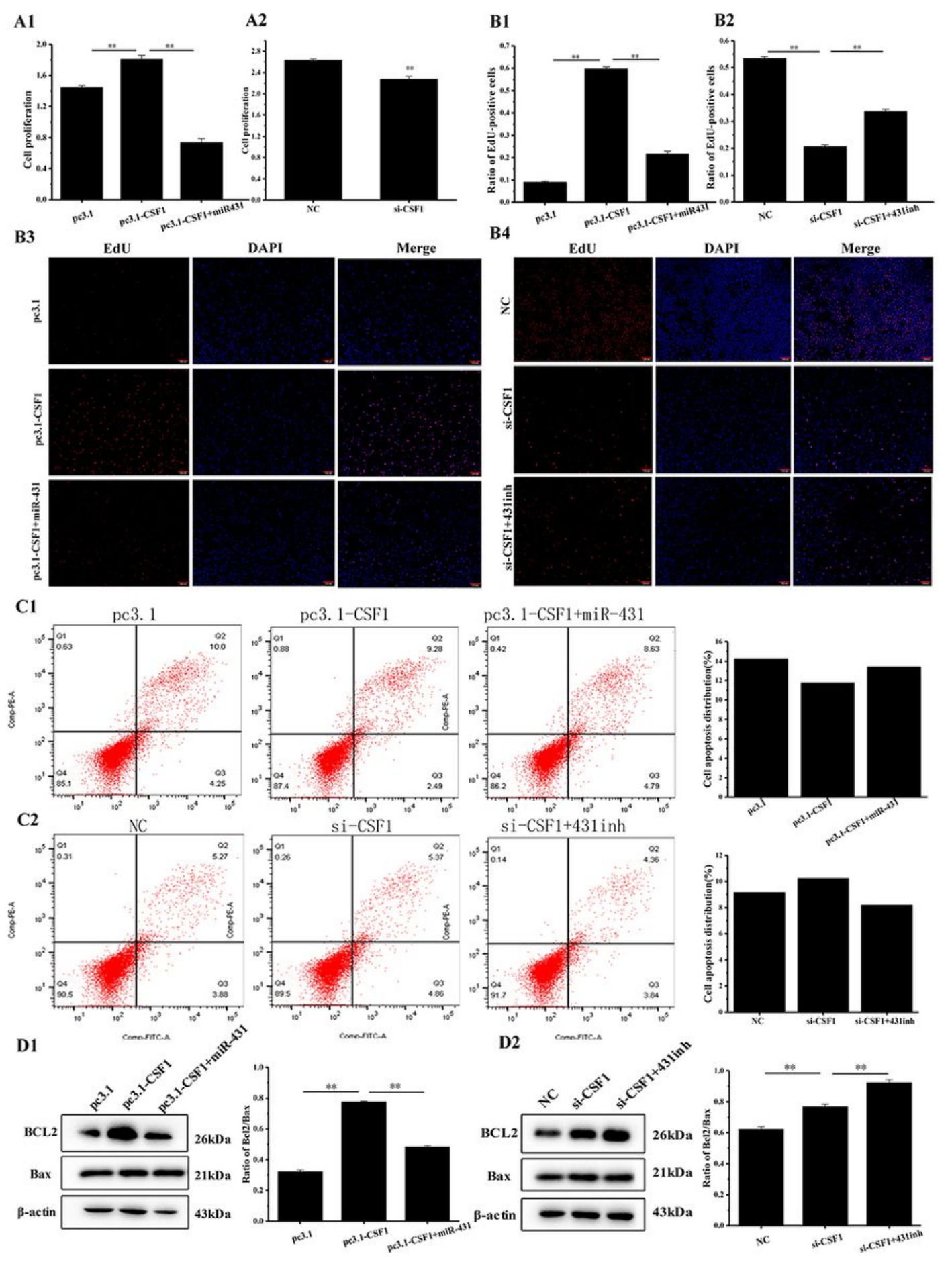

Figure 3

CSF1 promoted cell proliferation in GEECs. A: Cell proliferation was detected by CCK-8, B: Cell proliferation was detected by EdU, the scale bar was $200 \mu \mathrm{m}$. C: Cell apoptosis was detected by flow cytometry, D: Protein level was detected by Western blot, $\beta$-actin was used as a loading control. Date are expressed as mean $\pm S E, n=3$. * denotes that the difference is significant $(P<0.05)$, ** denotes that the difference is very significant $(P<0.01)$. 

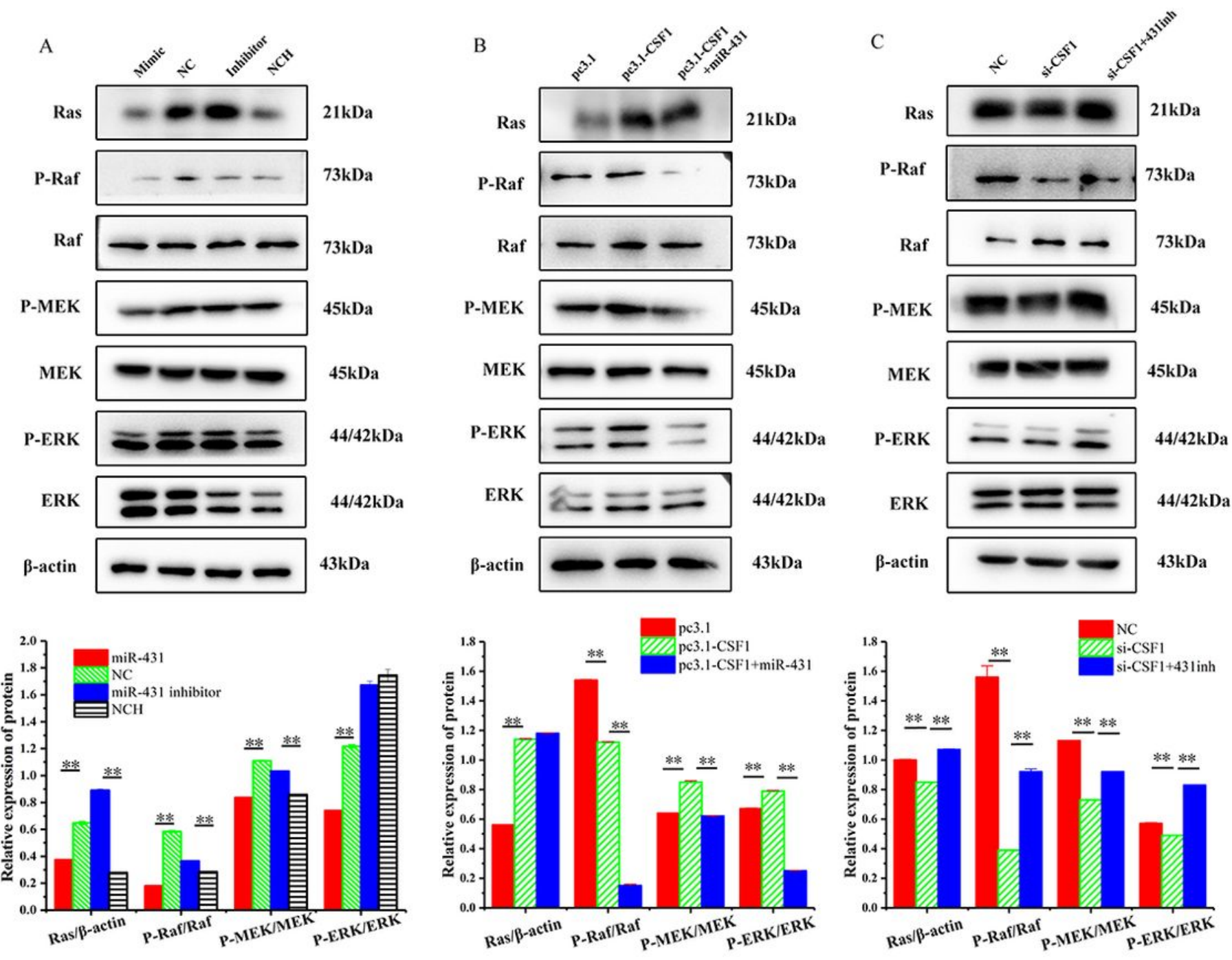

\section{Figure 4}

Effects of miR-431 on MAPK signaling pathway through CSF1. A-C: Protein level was detected by Western blot, $\beta$ actin was used as a loading control. Date are expressed as mean $\pm S E, n=3$. * denotes that the difference is significant $(P<0.05)$, ** denotes that the difference is very significant $(P<0.01)$. 


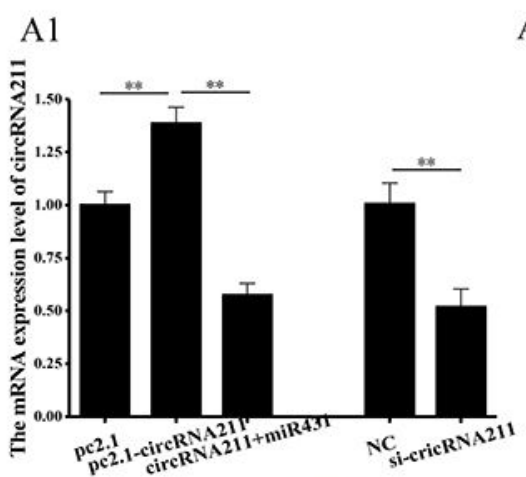

D1

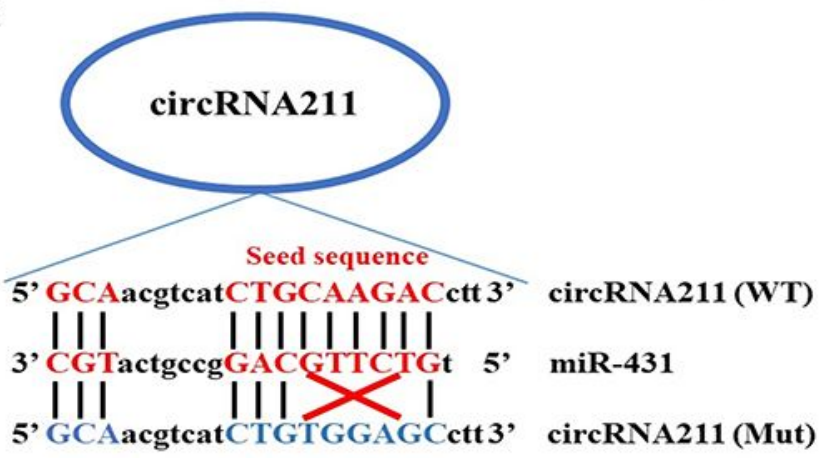

F1

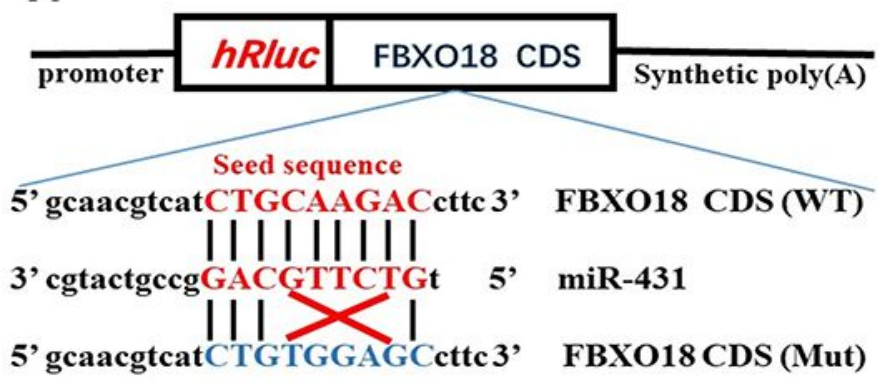

B

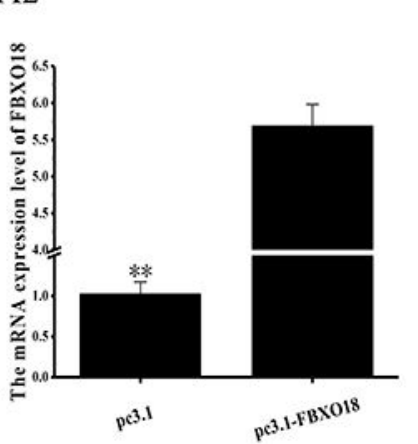

D2
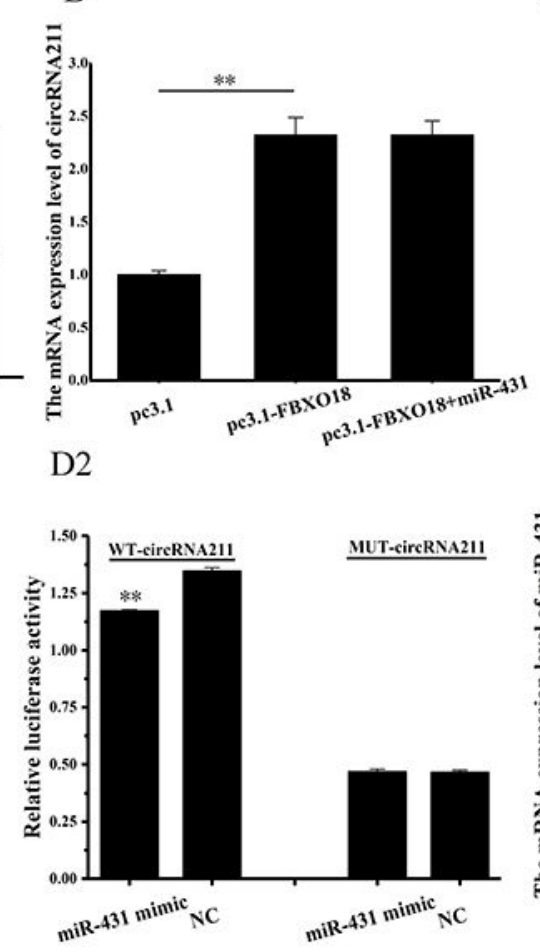

F2

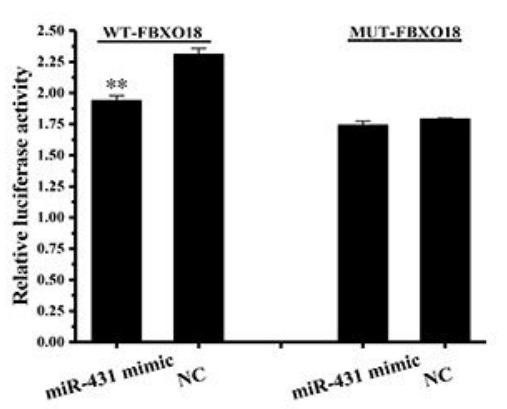

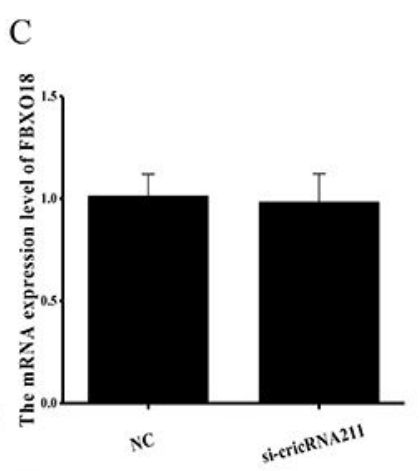

$\mathrm{E}$
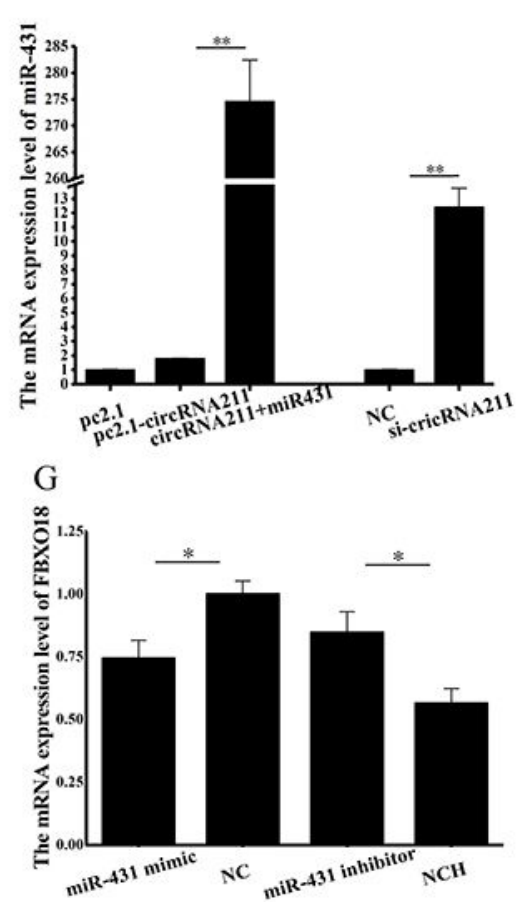

\section{Figure 5}

circRNA211 competitively inhibited miR-431 while miR-431 targeted FBX018 in GEECs. A: Transfection efficiency of circRNA211 and FBX018, B: The expression level of circRNA211 was detected by RT-qPCR. C: The expression level of FBX018 was detected by RT-qPCR. D1: Schematic diagram for construction of wild-type and mutant dual luciferase reporter vectors. The nucleotide sequence marked in red is the seed sequence that miR-431 binds to circRNA211, while the nucleotide sequence marked in blue is the mutant sequence. D2: Detection of dual luciferase activity. E: miR-431 expression level was detected by RT-qPCR. F1: Schematic diagram for construction of wild-type and mutant dual luciferase reporter vectors. The nucleotide sequence marked in red is the seed sequence that miR-431 binds to FBX018, while the nucleotide sequence marked in blue is the mutant sequence. F2: Detection of dual luciferase activity. G: FBX018 mRNA level was detected by RT-qPCR. Date are expressed as mean $\pm S E, n=3$. * denotes that the difference is significant $(P<0.05)$, ** denotes that the difference is very significant $(P<0.01)$. 
A1

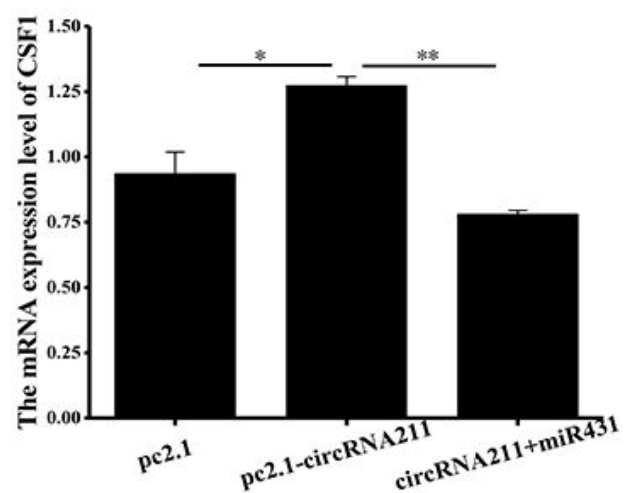

A2
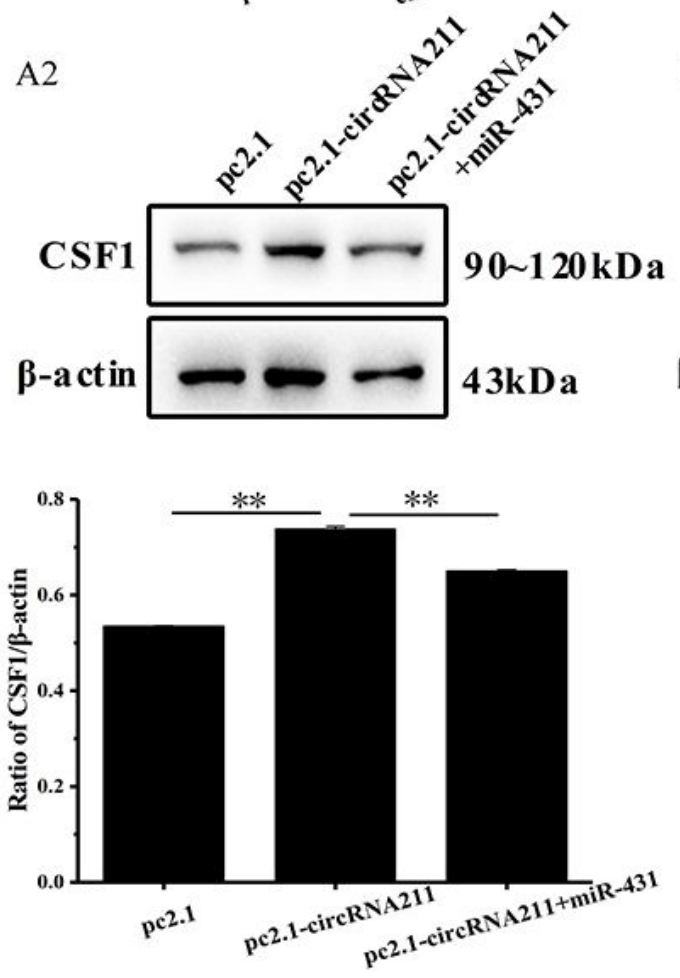

B1

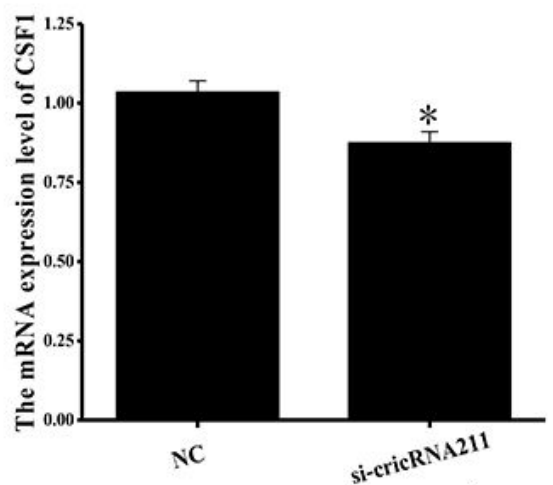

B2

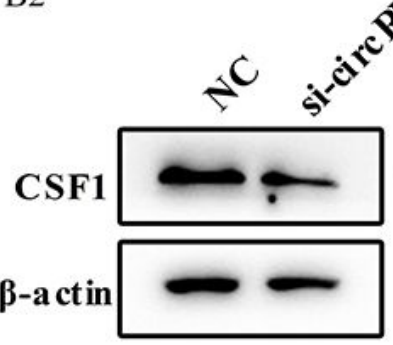

$43 \mathrm{kDa}$
C1

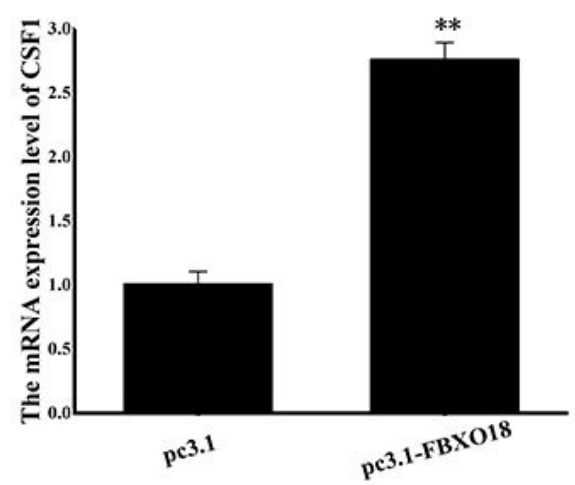

C2

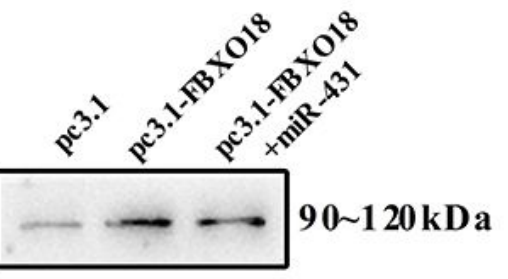

$\beta$-actin

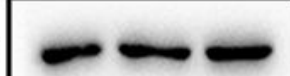

$43 \mathrm{kDa}$
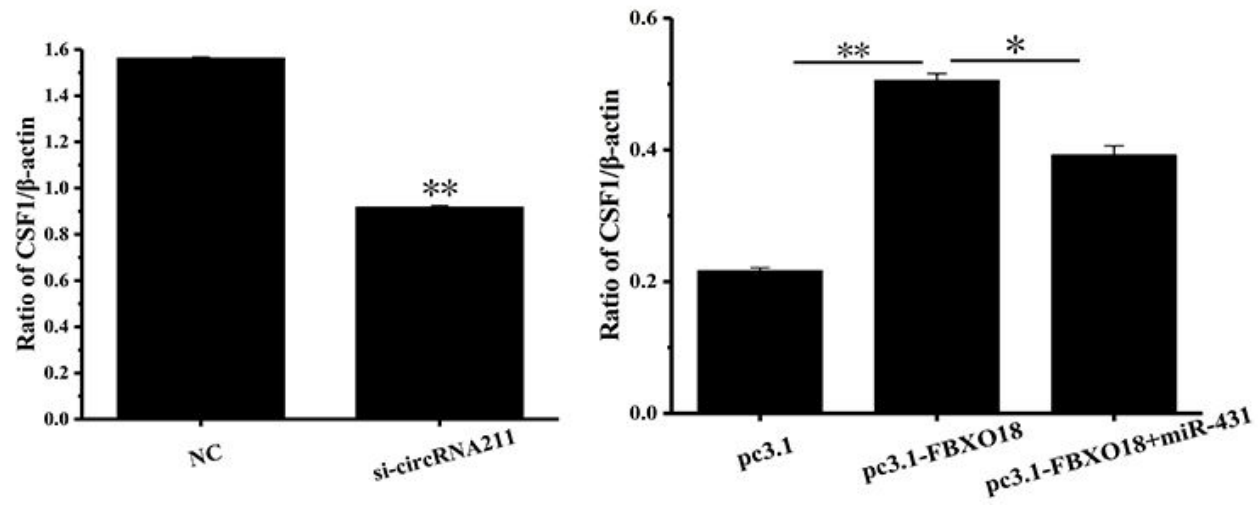

Figure 6

Effects of circRNA211 and FBX018 on CSF1 expression. A: circRNA211 promoted CSF1 expression at mRNA and protein levels. B: si-circRNA211 inhibited CSF1 expression at mRNA and protein levels, C: FBX018 promoted CSF1 expression at mRNA and protein levels, $\beta$-actin was used as a loading control. Date are expressed as mean $\pm S E$, $\mathrm{n}=3$. * denotes that the difference is significant $(\mathrm{P}<0.05)$, ** denotes that the difference is very significant $(\mathrm{P}<$ $0.01)$. 

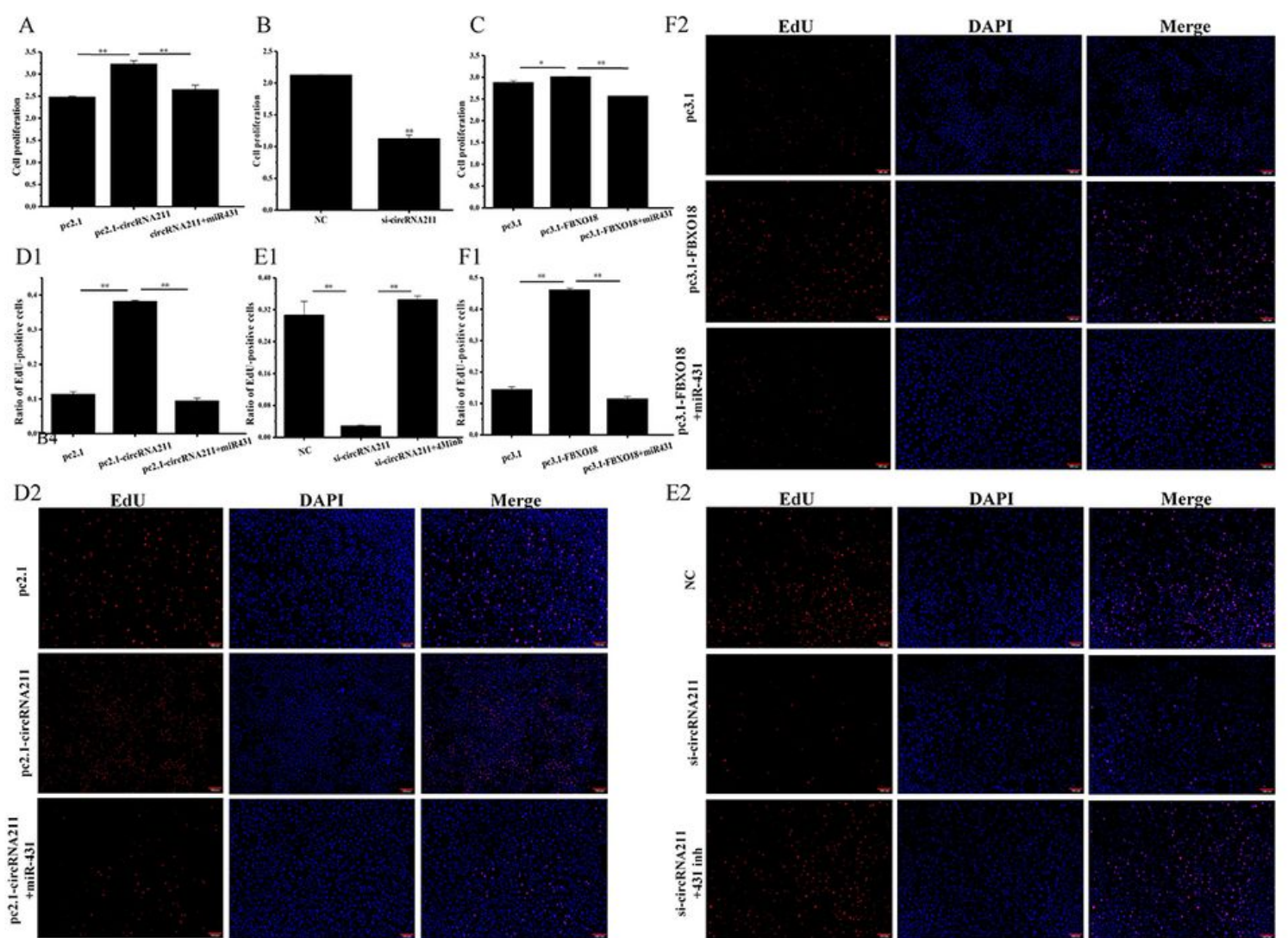

E2
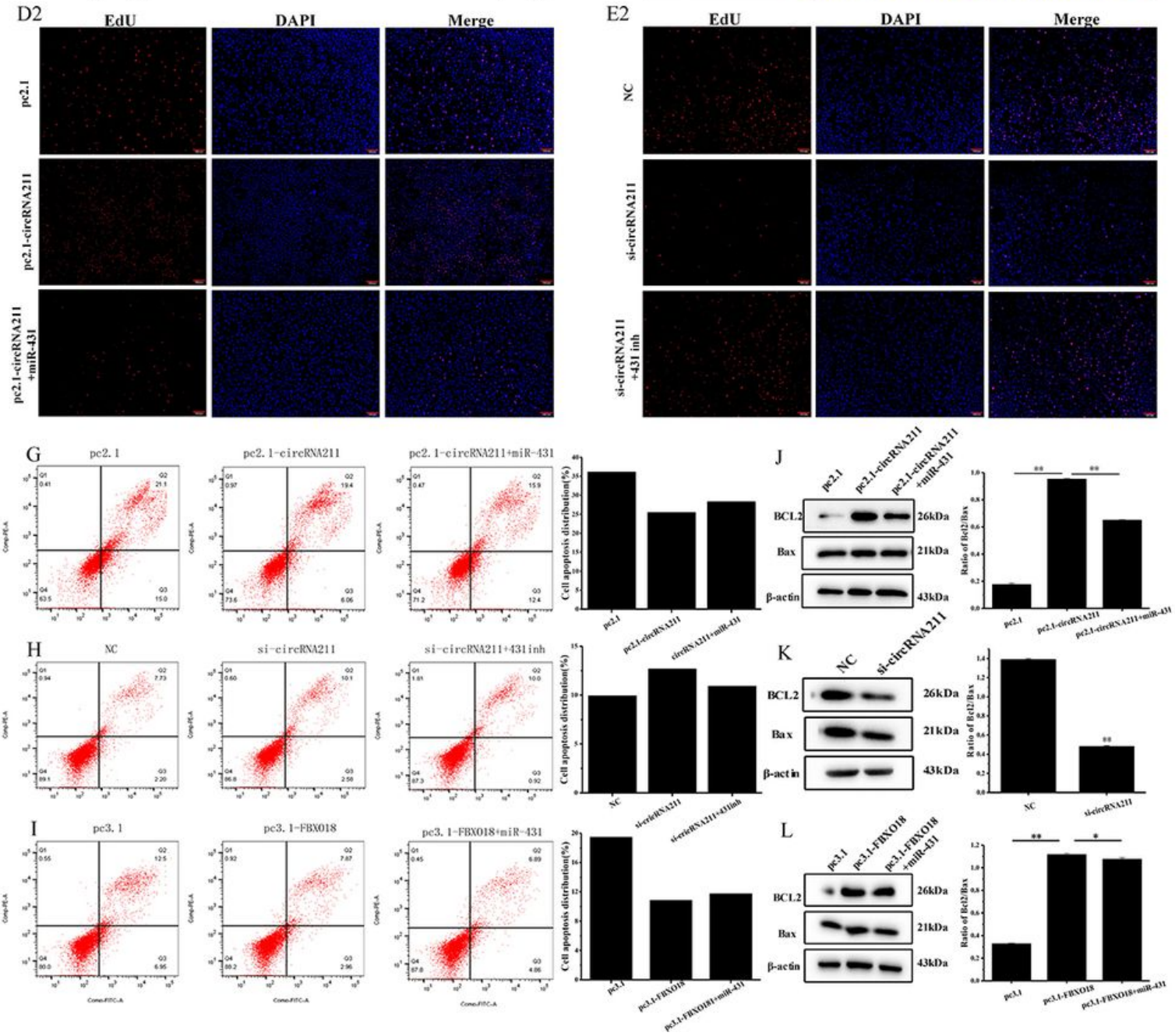

\section{Figure 7}

circRNA211 and FBX018 promoted the proliferation of GEECs. A, B, C: Cell proliferation was detected by CCK-8, D, E, F: Cell proliferation was detected by EdU, the scale bar was $200 \mu \mathrm{m}, \mathrm{G}, \mathrm{H}, \mathrm{I}$ : Cell apoptosis was detected by flow cytometry, J, K, L: Protein level was detected by Western blot, $\beta$-actin was used as a loading control. Date are expressed as mean $\pm S E, n=3$. * denotes that the difference is significant $(P<0.05)$, ** denotes that the difference is very significant $(P<0.01)$. 

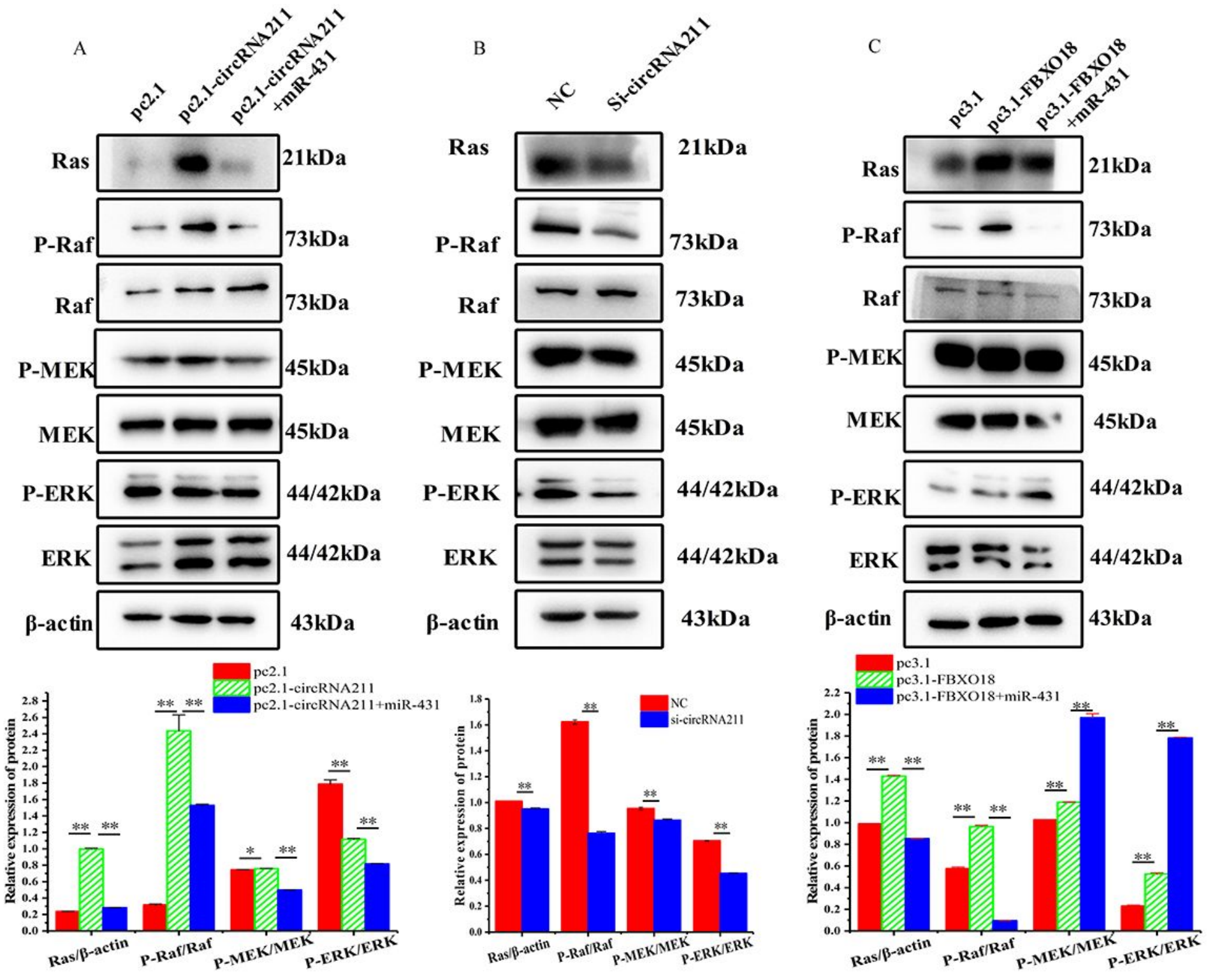

Figure 8

circRNA211 and FBX018 activated the MAPK pathway by inhibiting miR-431. A-C: Protein level was detected by Western blot, $\beta$-actin was used as a loading control. Date are expressed as mean $\pm S E, n=3$. * denotes that the difference is significant $(P<0.05)$, ** denotes that the difference is very significant $(P<0.01)$. 

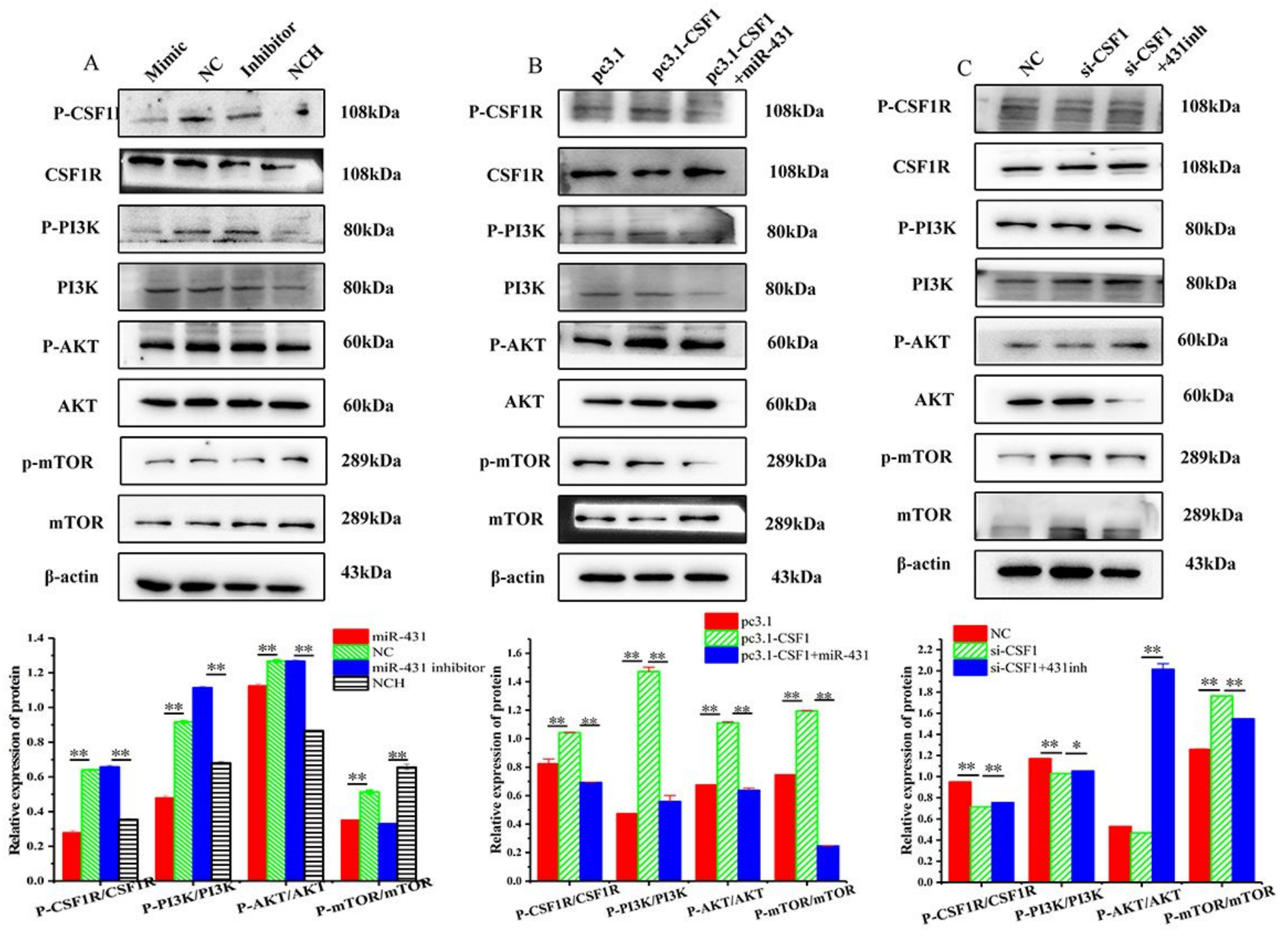

\section{Figure 9}

miR-431 inhibited CSF1R/PI3K/AKT signaling pathway through CSF1. A-C: Protein level was detected by Western blot, $\beta$-actin was used as a loading control. Date are expressed as mean $\pm S E, n=3$. * denotes that the difference is significant $(P<0.05)$, ** denotes that the difference is very significant $(P<0.01)$. 


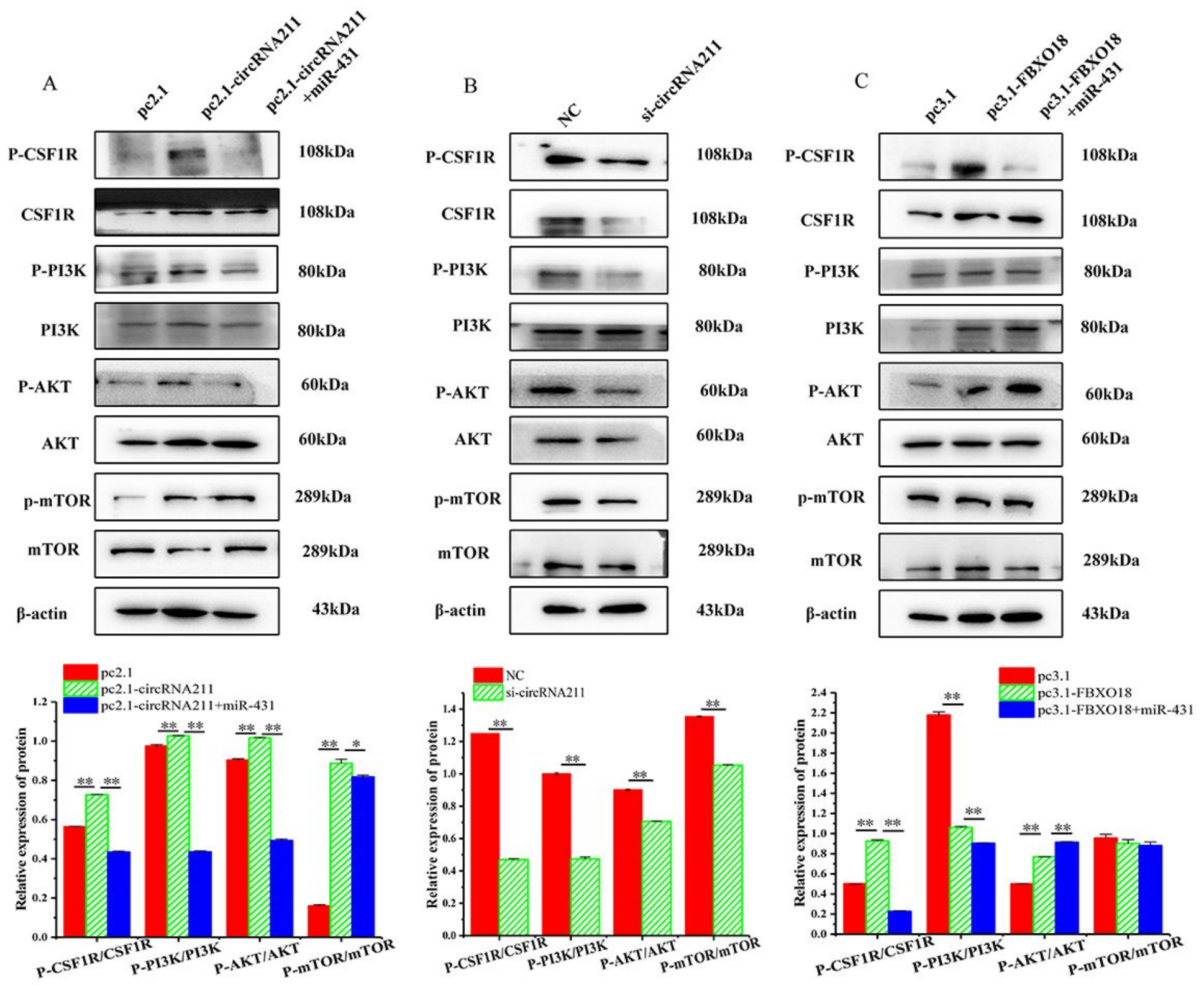

\section{Figure 10}

circRNA211 activated the CSF1R/PI3K/AKT pathway through miR-431. A-C: Protein level was detected by Western blot, $\beta$-actin was used as a loading control. Date are expressed as mean $\pm S E, n=3$. * denotes that the difference is significant $(P<0.05)$, ** denotes that the difference is very significant $(P<0.01)$. 
A

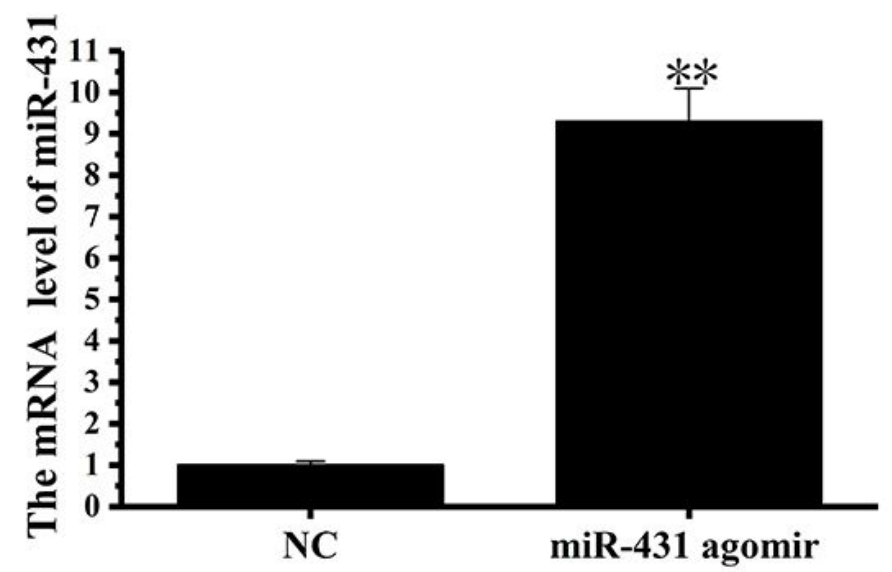

B

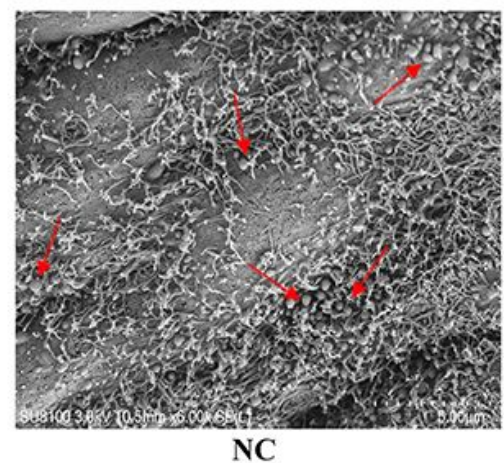

E

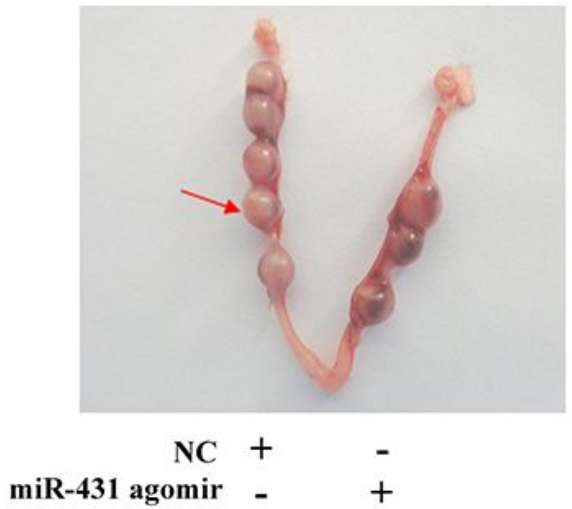

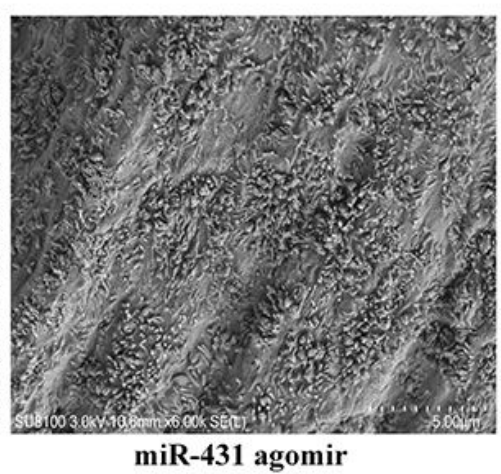

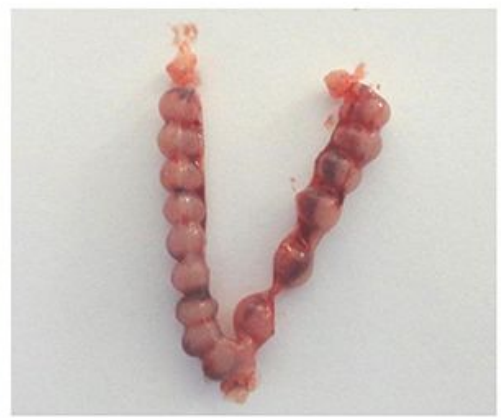

$+$

C
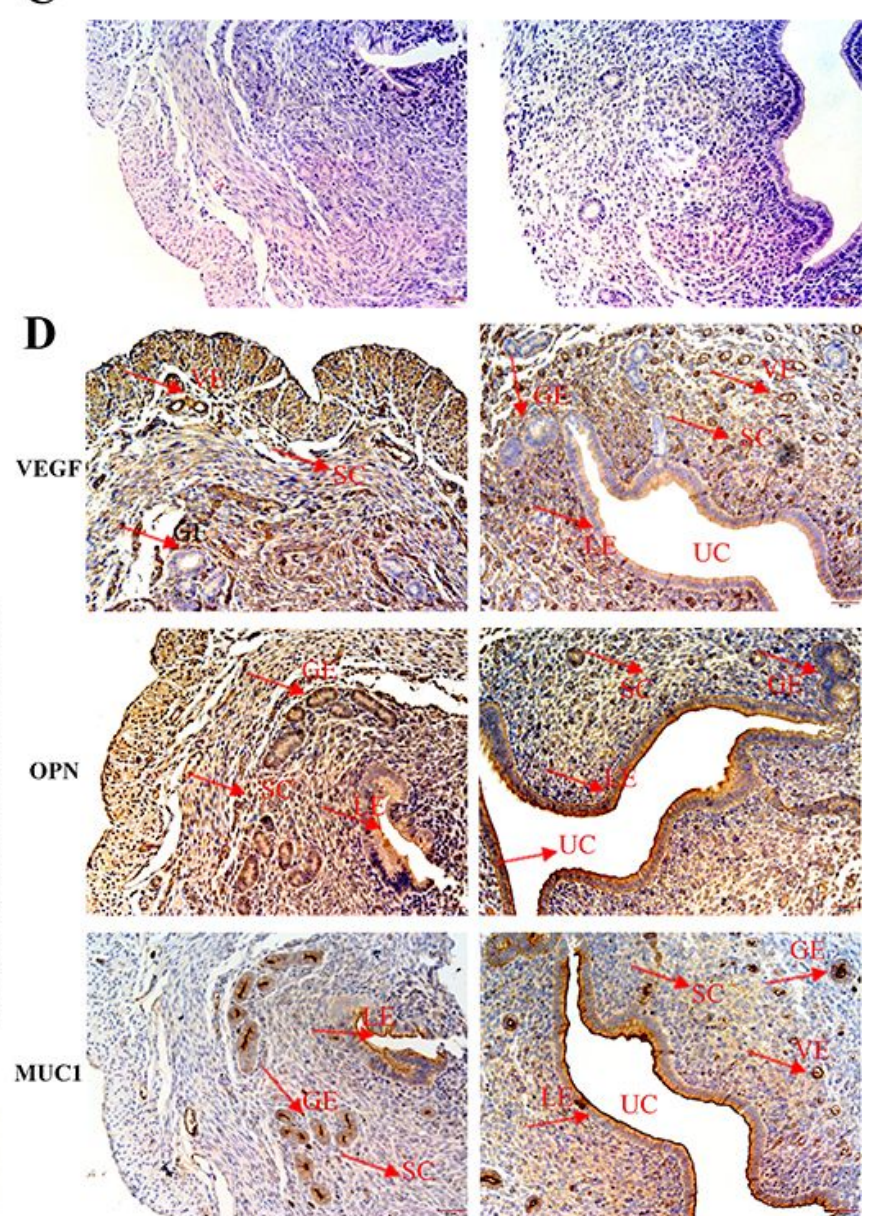

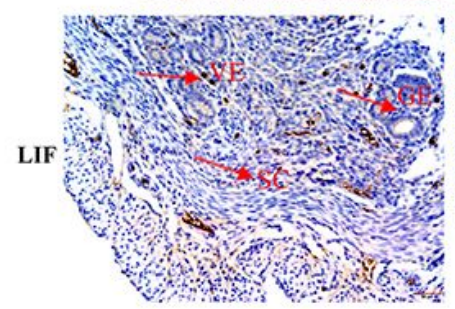

NC
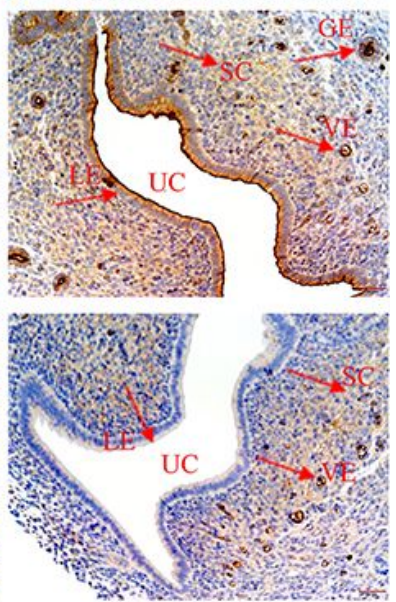

miR-431 agomir

\section{Figure 11}

Effect of miR-431 on the establishment of endometrial receptivity in mice. A: The mRNA expression level of miR431 was detected by RT-qPCR, B: The surface morphology of the endometrium was observed by scanning electron microscopy, the red arrows pointed to the pinopodes, C: The thickness of the endometrium was observed by HE staining, the scale bar was $50 \mu \mathrm{m}, \mathrm{D}$ : Immunohistochemistry was used to detect the protein expression of the marker genes in the endometrium, UC: uterine cavity, LE: luminal epithelial cells, GE: glandular epithelial cells, SC: stromal cells, VE: vascular epithelial cells. The red scale is $50 \mu \mathrm{m}, \mathrm{E}: \mathrm{miR}-431$ inhibited mice embryo implantation. Date are expressed as mean $\pm S E, n=3$. 